Métodos de contagem

Marcelo Henrique Trovão 
SERVIÇO DE PÓS-GRADUAÇÃO DO ICMC-USP

Data de Depósito:

Assinatura:

\section{Métodos de contagem}

\section{Marcelo Henrique Trovão}

Orientador: Prof. Dr. Hermano de Souza Ribeiro

Dissertação apresentada ao Instituto de Ciências Matemáticas e de Computação - ICMC-USP, como parte dos requisitos para obtenção do título de Mestre

- Programa de Mestrado Profissional em Matemática. VERSÃO REVISADA

USP - São Carlos

Maio de 2015 
Ficha catalográfica elaborada pela Biblioteca Prof. Achille Bassi e Seção Técnica de Informática, ICMC/USP, com os dados fornecidos pelo(a) autor(a)

T843m Trovão, Marcelo Henrique
Métodos de contagem / Marcelo Henrique Trovão;
orientador Hermano de Souza Ribeiro. -- São
Carlos, 2015.
67 p.
Dissertação (Mestrado - Programa de Pós-Graduação
em Mestrado Profissional em Matemática em Rede
Nacional) -- Instituto de Ciências Matemáticas e de
Computação, Universidade de São Paulo, 2015.
1. Contagem indireta. 2. Princípio de inclusão e
exclusão. 3. Número de funções. 4. Permutação. I.
Ribeiro, Hermano de Souza, orient. II. Título.


Dedico esse trabalho a todos os amigos que fiz durante os estudos e à minha esposa, em especial, pelo apoio e confiança em mim depositada. 


\section{Agradecimentos}

Primeiramente a Deus, por permitir a oportunidade de conclusão do mestrado.

Ao orientador, Professor Dr. Hermano de Souza Ribeiro, pelo incentivo, paciência e dedicação durante a elaboração da dissertação.

Aos colegas de estudo, pela amizade e companheirismo.

Aos professores, Esther Pacheco de Almeida Prado, Ires Dias, Luiz Augusto da Costa Ladeira, Marcia Cristina Anderson Braz Federson, Miguel Vinicius Santini Frasson, Miriam Cardoso Utsumi, Oziride Manzoli Neto, Paulo Leandro Dattori da Silva, Sérgio Henrique Monari Soares, Sérgio Luis Zani, por todo o aprendizado. 


\section{Resumo}

Neste trabalho, estudamos alguns números e procedimentos que facilitam na solução de problemas no campo da contagem, sendo esses: O Princípio da Inclusão e Exclusão, Triângulo de Pascal, Binômios de Newton, números binomiais e multinomiais, números de funções, permutações, grafos, números de Stirling, lemas de Kaplansky e sequência de Fibonacci.

Palavras-chave: Contagem; Números; Recorrência; Stirling; Kaplansky; Fibonacci; Funções. 


\begin{abstract}
We studied some numbers and procedures that facilitate the solution of problems in the field of counting, these being: The Principle of Inclusion and Exclusion, Pascal's Triangle, Newton binomial, binomial and multinomial numbers, numbers of functions, permutations, graphs, Stirling numbers, slogans Kaplansky and the Fibonacci sequence.
\end{abstract}

Keywords: Counting; Numbers; Recurrence; Stirling; Kaplansky; Fibonacci; Functions. 


\section{Sumário}

1 INTRODUCÃ $\mathbf{O}$

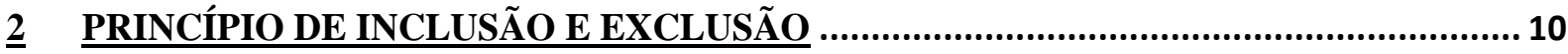

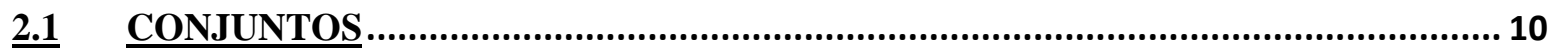

2.1.1 RELACÃO DE INCLUSÃO ENTRE CONJUNTOS................................................ 10

2.1.2 UNIÃO ENTRE CONJUNTOS ........................................................................................ 11

$\underline{\text { 2.1.3 }}$ INTERSECÃO DE CONJUNTOS ……………………................................................ 11

2.1.4 CONJUNTO COMPLEMENTAR ................................................................................. 12

2.1.5 DIFERENÇA ENTRE CONJUNTOS........................................................................... 13

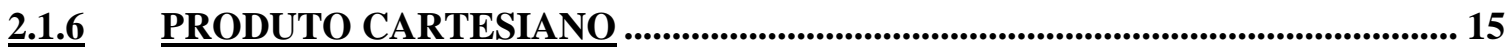

2.1.7 PARTIČÃO DE CONJUNTO......................................................................................... 16

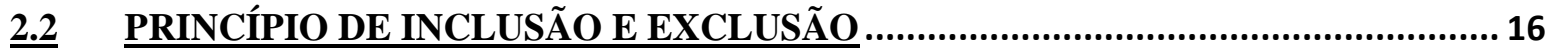

2.3 FUNCC̃̃O TOTIENTE DE EULER E O PRINCÍPIO DE INCLUSÃO-EXCLUSÃO. 20

$\underline{3}$ TRIÂNGULO DE PASCAL............................................................................................. 22

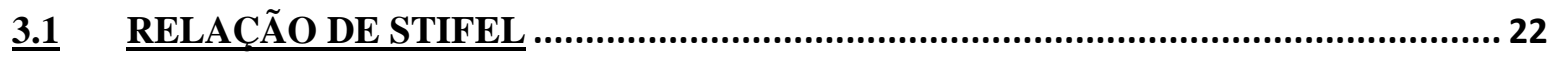

3.2 REGRA DA SOMA DE UMA LINHA …………................................................ 23

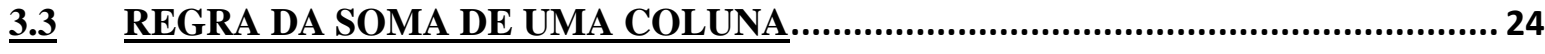

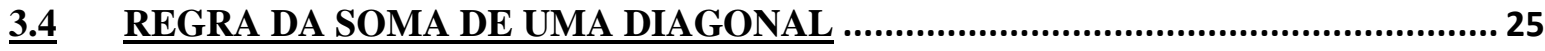

3.5 BINÔMIO DE NEWTON…………………………….............................................. 26

3.6 SEQUÊNCIA DE FIBONACCI E O TRIÂNGULO DE PASCAL ………………….... 28

$4 \quad$ RELACÃ O BINÁRIA.................................................................................................... 30

4.1 TIPOS DE RELACÕES BINÁRIAS.................................................................. 30

4.2 PROPRIEDADE DAS RELAC ÕES ………………......................................... 31

4.2.1 RELACÃO REFLEXIVA …………………………..................................................... 31

4.2.2 RELAÇ̃̃O SIMÉTRICA ....................................................................................... 32

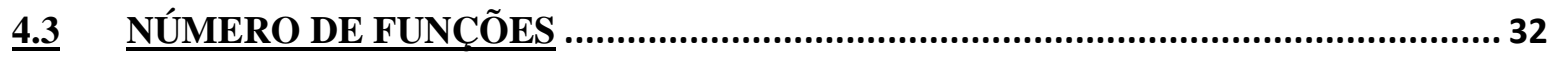

4.3.1 NÚMERO DE FUNCÕES INJETORAS ……………….............................................. 33

4.3.2 NÚMERO DE FUNCÕES BIJETORAS ………………........................................... 33

4.3.3 NÚMERO DE FUNCÕES SOBREJETORAS ……….............................................. 33

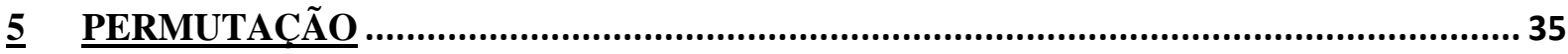

5.1 NÚMERO DE PERMUTACÕES SUJEITA A RESTRIC ÕES.................................... 36

5.2 NÚMERO DE FUNCÕES CRESCENTES OU DECRESCENTES ……..................... 39

$\underline{5.3}$ PERMUTACÕES CAÓTICAS............................................................................. 43 
5.4 NÚMEROS DE FUNCÕES COM RESTRICÕES ................................................... 47

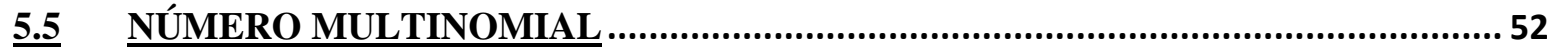

$\underline{5.6}$ NÚMEROS DE STIRLING DE PRIMEIRA ESPÉCIE ......................................... 54

5.7 NÚMEROS DE STIRLING DE SEGUNDA ESPÉCIE ….......................................57

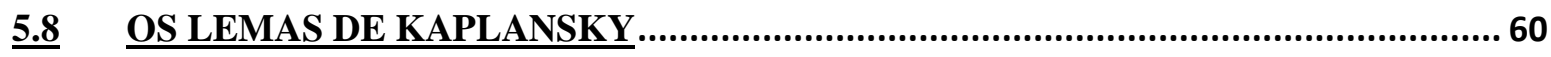

5.9 APLICACÕES PARA A SEQUÊNCIA DE FIBONACCI …...................................63

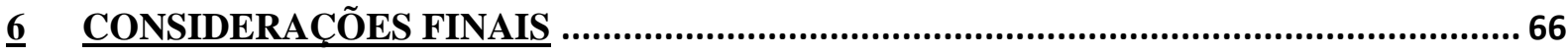

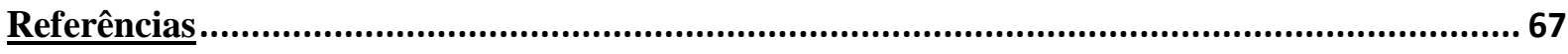




\section{INTRODUÇÃO}

O objetivo deste trabalho é expor aos alunos e professores do ensino médio alguns números e procedimentos que são úteis na solução de problemas no campo da contagem, em que tais procedimentos e números dificilmente são usados nas aulas de matemática. De acordo com o documento dos Parâmetros Curriculares Nacionais (PCN), destaca-se a importância do desenvolvimento do raciocínio combinatório com os alunos do ensino médio.

Técnicas e raciocínios estatísticos e probabilísticos são, sem dúvida, instrumentos tanto das ciências da Natureza quanto das Ciências Humanas. Isto mostra como será importante uma cuidadosa abordagem dos conteúdos de contagem, estatística e probabilidades no Ensino Médio, ampliando a interface entre o aprendizado da Matemática e das demais ciências e áreas (BRASIL, 2000).

A motivação para a escolha desse tema é a dificuldade que alguns problemas de contagem apresentam e que apenas o conhecimento dos princípios aditivo e multiplicativo torna-se, quase sempre, insuficiente para a solução, sendo necessário, então, o desenvolvimento ou o aprofundamento de novas ideias. Não faz parte deste estudo esgotar todos os procedimentos de contagem existentes e sim servir como estimulo à curiosidade e incentivo a novas pesquisas.

Como abordagem inicial, no primeiro capítulo temos a notação básica utilizada no desenvolvimento da pesquisa, e o Princípio de Inclusão e Exclusão, que servirá para resolver alguns problemas, sendo útil também na fundamentação de novas ideias, como a obtenção do número de funções sobrejetoras e a obtenção da fórmula da função $\Phi$ de Euler.

O capítulo 2 contém o Triângulo de Pascal, a Relação de Stifel, os Binômios de Newton e suas aplicações em alguns problemas de genética, por meio da observação dos coeficientes e dos expoentes em sua expansão.

O capítulo 3 trata das relações binárias, os tipos de relações (total, sobrejetoras, funcional), algumas propriedades, relações reflexivas e simétricas, a contagem do número de funções, injetoras e sobrejetoras e algumas aplicações.

O último capítulo é destinado ao estudo das permutações, com destaque à aplicação das permutações caóticas do Polinômio-torre e de grafos na solução de permutações com restrições, a obtenção do número de funções crescentes ou decrescentes, o número multinomial e a expansão multinomial de $(x+y+z)^{n}$, os números de Stirling de primeira e de segunda espécie, os lemas de Kaplansky e algumas aplicações para a sequência de Fibonacci. 


\section{PRINCÍPIO DE INCLUSÃO E EXCLUSÃO 2.1 CONJUNTOS}

Esse capítulo terá início com as notações e operações básicas entre conjuntos que aqui serão utilizadas.

Os conjuntos serão indicados por letras maiúsculas, como $A, B, C, \ldots, X, Y, Z$. Para indicar conjunto universo será utilizada a letra $U$ e no caso dos elementos usaremos letras minúsculas, como $a, b, c, \ldots, x, y, z$.

A relação de pertinência será representada pelo símbolo $\in$ e quando quisermos indicar que determinado elemento não pertence a um conjunto $A$, escrevemos $m \notin A$ (lê-se m não pertence ao conjunto A).

Se um conjunto tiver um número reduzido de elementos, a representação será conjunto seguido do símbolo de igual os seus elementos entre chaves e separados por vírgula, por exemplo, $A=\{1,2,3\}$. Para o conjunto vazio usaremos o símbolo $\emptyset$. Um conjunto poderá ser indicado por uma determinada propriedade ou característica de seus elementos como, $I=\{x / x=2 n+1, n=1,2,3, \ldots\}$ (lê-se $\mathrm{x}$, tal que $\mathrm{x}$ igual a $2 \mathrm{n}+1$ com $\mathrm{n}$ natural).

A cardinalidade de um conjunto será representada por duas barras laterais, exemplo $|A|=3$, que indica que o conjunto $A$ possui três elementos.

\subsubsection{RELAÇÃO DE INCLUSÃO ENTRE CONJUNTOS}

Se todo elemento de um conjunto A também é elemento de um conjunto B então dizemos que o conjunto A está contido em $\mathrm{B}$ e representamos por $A \subset B$ ou $B \supset A$.

A figura 1.1 ilustra a situação.

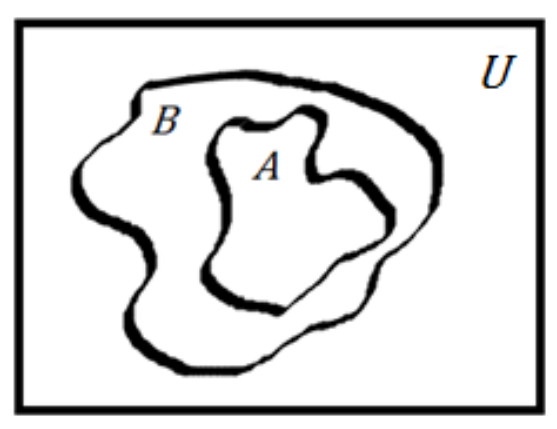

Fig. 1.1 
Se $A \subset B$ ou $B \subset A$ então $A=B$.

\subsubsection{UNIÃO ENTRE CONJUNTOS}

O conjunto união $A \cup B$ de dois conjuntos $A$ e $B$ é definido por

$$
\{x \in U / x \in A \text { ou } x \in B\}
$$

por exemplo, $A=\{m, a, c\}, B=\{t, e, i\}$ e $A \cup B=\{m, a, t, e, i, c\}$.

A parte cinza da figura 1.2 ilustra o conjunto $A \cup B$.

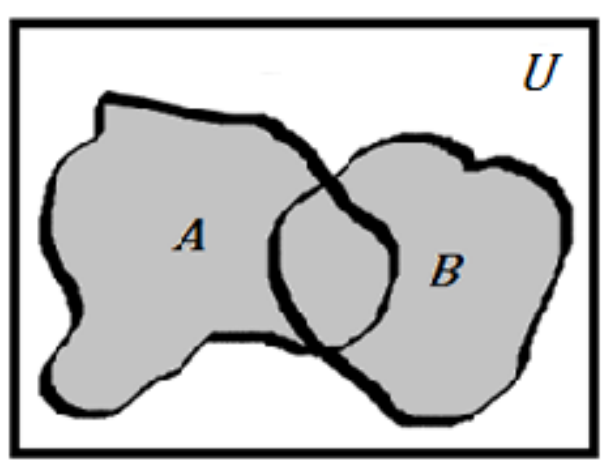

Fig. 1.2

A união de $n$ conjuntos, $A_{1}, A_{2}, A_{3}, \ldots, A_{n}$, é definida analogamente e representada por

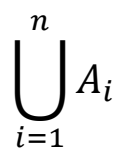

\subsubsection{INTERSEÇÃO DE CONJUNTOS}

O conjunto interseção $A \cap B$ de dois conjuntos $A$ e $B$ é definido por

$$
\{x \in U / x \in A \text { e } x \in B\}
$$

Dados os conjuntos $A$ e $B$, o conjunto interseção é o conjunto formado pelos elementos contidos simultaneamente em $A$ e $B$, por exemplo, $A=\{m, a, t, e\}, B=$ $\{m, a, t, i, c\}, A \cap B=\{m, a, t\}$. 
A área cinza da figura 1.3 representa a interseção entre $A$ e $B$.

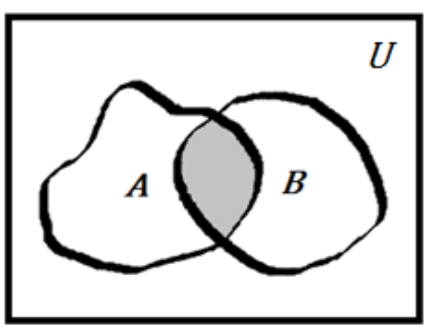

Fig.1.3

Para o caso geral de $n$ conjuntos, $A_{1}, A_{2}, A_{3}, \ldots, A_{n}$, definimos analogamente e representamos por

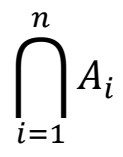

Se a interseção de dois conjuntos $A$ e $B$ é tal que, $A \cap B=\emptyset$, então esses conjuntos são disjuntos. A figura 1.4 ilustra essa situação.

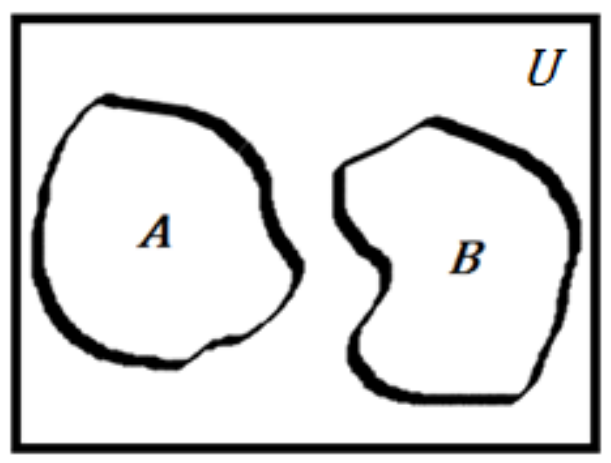

Fig. 1.4

\subsubsection{CONJUNTO COMPLEMENTAR}

O conjunto complementar $A^{c}$ de um conjunto $A$ é definido por

$$
\{x \in U / x \notin A\}
$$

O complementar do conjunto $A$ é o conjunto formado por todos os elementos de $U$ que não pertencem ao conjunto $A$, ou ainda, é o conjunto que completaria $A$ para que fosse igual a $U$. 
A área cinza da figura 1.5 representa o complementar de $A$.

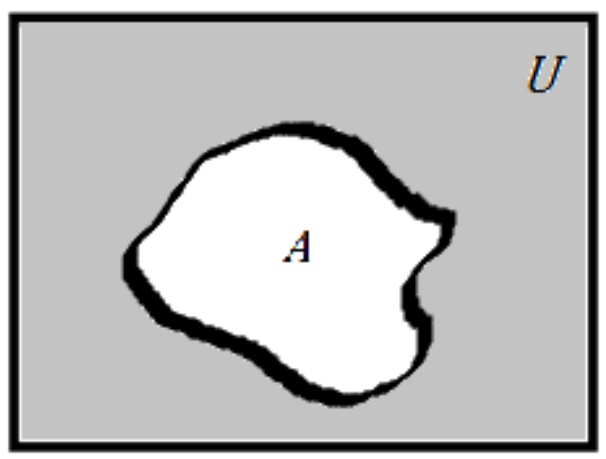

Fig. 1.5

\subsubsection{DIFERENÇA ENTRE CONJUNTOS}

O conjunto diferença $A-B$ entre dois conjuntos $A$ e $B$ é definido por

$$
A \cap B^{c}=\{x \in U / x \in A \quad e \quad x \notin B\}
$$

A área cinza da figura 1.6 ilustra a diferença entre os conjuntos $A$ e $B$.

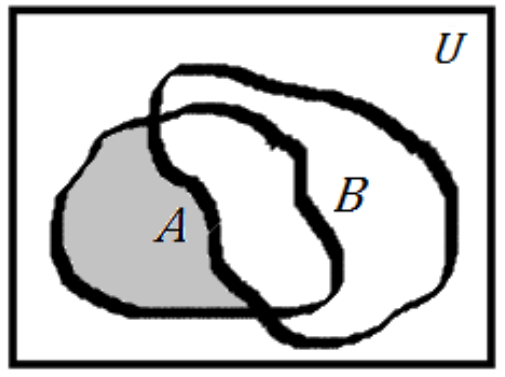

Fig. 1.6

\section{Teorema 1.}

1. Para todo conjunto $A \subset U, A \cup \emptyset=A, A \cap \emptyset=\emptyset$.

2. $A \subset B$, se e somente se, $A \cup B=B$.

3. $A \subset B$, se e somente se, $A \cap B=A$.

4. $A \cup(B \cup C)=(A \cup B) \cup C$ (Associativa). 
Por definição:

$A \cup(B \cup C)=\{x \in A$ ou $\boldsymbol{x} \in(\boldsymbol{B} \cup \boldsymbol{C})\}$.

$\boldsymbol{x} \in(\boldsymbol{B} \cup \boldsymbol{C})=\{x \in B$ ou $x \in C\}$, logo:

$A \cup(B \cup C)=\{x \in A$ ou $x \in B$ ou $x \in C\}=(A \cup B) \cup C$

5. $A \cap(B \cap C)=(A \cap B) \cap C$ (Associativa).

Por definição:

$A \cap(B \cap C)=\{x \in A$ e $x \in(B \cap C)\}$.

$\boldsymbol{x} \in(\boldsymbol{B} \cap \boldsymbol{C})=\{x \in B$ e $x \in C\}$, logo:

$$
A \cap(B \cap C)=\{x \in A, x \in B \text { e } x \in C\}=(A \cap B) \cap C
$$

6. $A \cap(B \cup C)=(A \cap B) \cup(A \cap C)$ (distributiva).

Por definição:

$A \cap(B \cup C)=\{x \in A$ e $x \in(B \cup C)\}$.

Então, ou $x \in A$ e $B$, ou $x \in A$ e $C$, dessa forma:

$$
(A \cap B) \cup(A \cap C)=A \cap(B \cup C)
$$

7. $A \cup(B \cap C)=(A \cup B) \cap(A \cup C)$ (distributiva).

Por definição:

$A \cup(B \cap C)=\{x \in A$ ou $\boldsymbol{x} \in(\boldsymbol{B} \cap \boldsymbol{C})\}$.

$\boldsymbol{x} \in(\boldsymbol{B} \cap \boldsymbol{C})=\{x \in B$ e $x \in C\}$, assim:

$$
A \cup(B \cap C)=\{x \subset(A \cup B) \text { e } x \subset(A \cup C)\}=(A \cup B) \cap(A \cup C)
$$

8. $A \cup A^{c}=U, A \cap A^{c}=\emptyset$.

9. $\left(A^{c}\right)^{c}=A ; A \subset B$, se e somente se, $B^{c} \subset A^{c}$.

Leis de Augustus De Morgan

10. $(A \cup B)^{c}=A^{c} \cap B^{c}$.

Por definição:

$A \cup B=\{x \in U / x \in A$ ou $x \in B\}$.

Dessa forma $(A \cup B)^{c}=\{x \in U / x \notin A$ e $x \notin B\}=A^{c} \cap B^{c}$ 
A figura 1.7 ilustra essa situação.

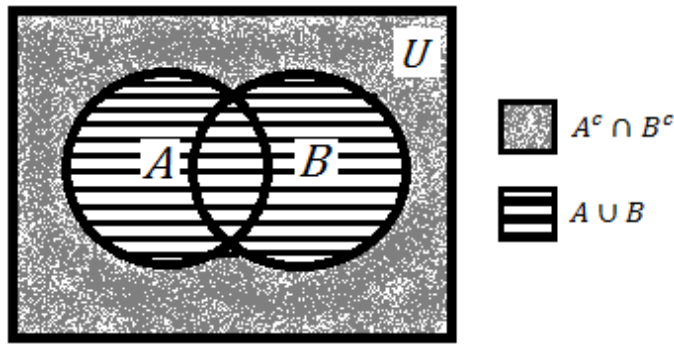

Fig. 1.7

11. $(A \cap B)^{c}=A^{c} \cup B^{c}$.

Por definição:

$A \cap B=\{x \in U / x \in A$ e $x \in B\}$.

Dessa forma $(A \cap B)^{c}=\{x \in U / x \notin A$ ou $x \notin B\}=A^{c} \cup B^{c}$

A figura 1.8 ilustra essa situação.

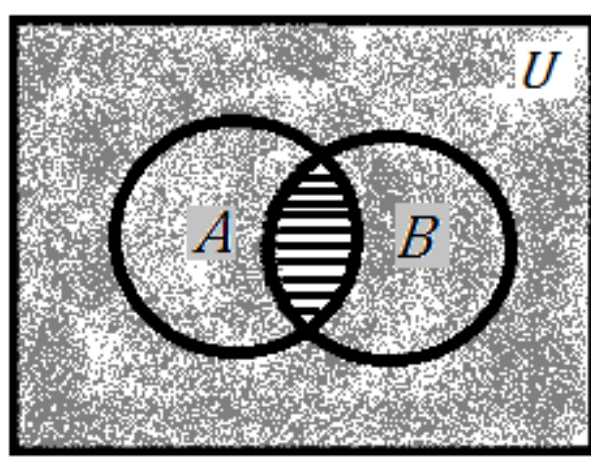

$A^{c} \cup B^{c}$

Fig. 1.8

\subsubsection{PRODUTO CARTESIANO}

O produto cartesiano $A \times B$ de dois conjuntos $A$ e $B$ é definido por

$$
A \times B=\{(x, y) / x \in A \text { e } y \in B\}
$$

Sejam dois conjuntos $A$ e $B$, o produto cartesiano entre eles $A \times B$ é conjunto de pares ordenados (x, y) tal que $x \in A$ e $y \in B$. Simbolicamente

Exemplo: $A=\{1,2\}$ e $B=\{1,2,3\}$

$$
A \times B=\{(1,1) ;(1,2) ;(1,3) ;(2,1) ;(2,2) ;(2,3)\}
$$


De modo geral, o produto cartesiano de $n$ conjuntos $A_{1}, A_{2}, A_{3}, \ldots, A_{n}$ é o conjunto das $n$-uplas $\left(a_{1}, a_{2}, a_{3}, \ldots, a_{n}\right)$ em que $a_{1} \in A_{1}, a_{2} \in A_{2}, \ldots, a_{n} \in A_{n}$.

\subsubsection{PARTIÇÃO DE CONJUNTO}

Seja $A$ um conjunto finito não-vazio. Uma partição de $A$ é uma coleção $A_{1}, A_{2}, A_{3}, \ldots, A_{n}$ de subconjuntos não vazios de $A$ tais que:

1) $A_{1} \cup A_{2} \cup A_{3} \ldots \cup A_{n}=A$

2) $A_{i} \cap A_{j}=\emptyset$ se $i \neq j$, ou seja, disjuntos dois a dois.

Exemplo 1: Se $A=\{1,2,3,4\}$, então, $A=\left\{A_{1}, A_{2}, A_{3}\right\}$, em que $A_{1}=\{1\}, A_{2}=\{2\}$ e $A_{3}=\{3,4\}$, é uma partição de $A$.

\subsection{PRINCÍPIO DE INCLUSÃO E EXCLUSÃO}

O principio da inclusão e exclusão trata de uma forma de contar o número de elementos da união entre conjuntos disjuntos ou não. Em seu modelo mais simples, para dois conjuntos $A$ e $B$ temos que:

$$
|A \cup B|=|A|+|B|-|A \cap B|
$$

O principio da inclusão e exclusão para dois conjuntos $A$ e $B$ afirma que o número de elementos da união é igual ao número de elemento de $A$ somado aos de $B$ menos o número de elementos comuns entre $A$ e $B$. É fácil compreender porque devemos subtrair $|A \cap B|$, para isso vamos imaginar a seguinte situação.

Suponhamos que existam $y$ elementos comuns em $A$ e $B$, além disso, há $x$ elementos que pertencem exclusivamente a $A$ e $z$ elementos que aparecem apenas em $B$, dessa forma:

$$
\begin{gathered}
|A \cup B|=x+y+z ; \\
|A \cup B|=|A|+|B|-|A \cap B|=(x+y)+(z+y)-y= \\
=x+y+z
\end{gathered}
$$


Caso contrário, teria contado em duplicidade os elementos comuns entre $A$ e $B$ (veja figura 1.9 que ilustra a situação).

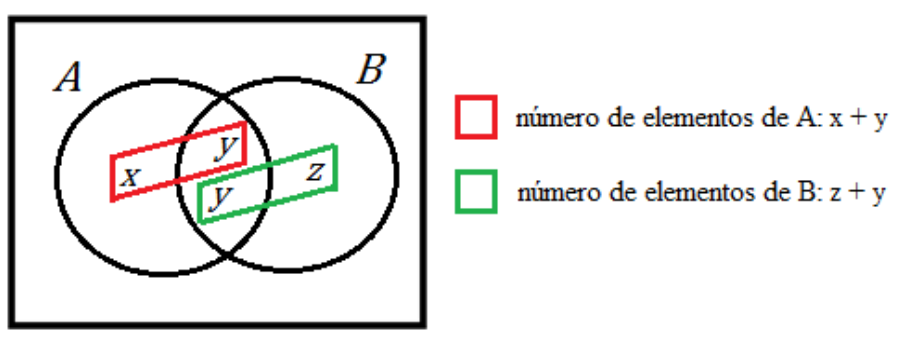

Fig. 1.9

Assim, $\left|(A \cup B)^{C}\right|=|U|-[|A|+|B|-|A \cap B|$

Para a união de três conjuntos $A, B$ e $C$ subconjuntos de $U$ temos que:

$$
|A \cup B \cup C|=|A|+|B|+|C|-|A \cap B|-|A \cap C|-|B \cap C|+|A \cap B \cap C|
$$

Podemos justificar com:

$$
\begin{gathered}
|A \cup B \cup C|=|A \cup(B \cup C)|=|A|+|B \cup C|-|A \cap(B \cup C)|= \\
=|A|+|B|+|C|-|B \cap C|-|A \cap(B \cup C)|= \\
=|A|+|B|+|C|-|B \cap C|-[|A \cap B| \cup|A \cap C|]= \\
=|A|+|B|+|C|-|B \cap C|-[|A \cap B|+|A \cap C|-|A \cap B \cap C|]= \\
=|A|+|B|+|C|-|A \cap B|-|A \cap C|-|B \cap C|+|A \cap B \cap C|
\end{gathered}
$$

Dessa forma:

$$
\begin{gathered}
\left|A^{c} \cap B^{c} \cap C^{c}\right|=|U|-|A \cup B \cup C|= \\
=|U|-[|A|+|B|+|C|]+[|A \cap B|+|A \cap C|+|B \cap C|]-|A \cap B \cap C| \\
\left|A \cap B^{c} \cap C^{c}\right|=|A|-[|A \cap B|+|A \cap C|]+|A \cap B \cap C| \\
\left|A \cap B \cap C^{c}\right|=|A \cap B|-|A \cap B \cap C|
\end{gathered}
$$


Justifica-se, analogamente, para quatro conjuntos $A, B, C$ e $D$ subconjuntos de $U$.

$$
\begin{aligned}
& |A \cup B \cup C \cup D|=|(A \cup B \cup C) \cup D|=|A \cup B \cup C|+|D|-|(A \cup B \cup C) \cap D|= \\
& =|A|+|B|+|C|-|A \cap B|-|A \cap C|-|B \cap C|+|A \cap B \cap C|+|D| \\
& -[|A \cap D| \cup|B \cap D| \cup|C \cap D|]= \\
& =|A|+|B|+|C|-|A \cap B|-|A \cap C|-|B \cap C|+|A \cap B \cap C|+|D| \\
& -[|A \cap D|+|B \cap D|+|C \cap D|-|(A \cap D) \cap(B \cap D)| \\
& -|(A \cap D) \cap(C \cap D)|-|(B \cap D) \cap(C \cap D)| \\
& +|(A \cap D) \cap(B \cap D) \cap(C \cap D)|]= \\
& =|A|+|B|+|C|+|D|-|A \cap B|-|A \cap C|-|A \cap D|-|B \cap C|-|B \cap D|-|C \cap D| \\
& +|A \cap B \cap C|+|A \cap B \cap D|+|A \cap C \cap D|+|B \cap C \cap D| \\
& -|A \cap B \cap C \cap D|
\end{aligned}
$$

Em suma, obtém-se o número de elementos da união entre os conjuntos $A_{1}, A_{2}, A_{3}, \ldots, A_{n}$ contidos em $U$, com:

$$
\begin{gathered}
\left|\bigcup_{i=1}^{n}\right| A_{i}\left|=\sum_{1 \leq i \leq n}\right| A_{i}\left|-\sum_{1 \leq i_{1}<i_{2} \leq i_{n}}\right| A_{i_{1}} \cap A_{i_{2}}\left|+\sum_{1 \leq i_{1}<i_{2}<i_{3} \leq i_{n}}\right| A_{i_{1}} \cap A_{i_{2}} \cap A_{i_{3}} \mid-\cdots \\
+(-1)^{n-1}\left|A_{1} \cap A_{2} \cap \ldots \cap A_{n}\right|
\end{gathered}
$$

Em que:

$$
\sum_{1 \leq i_{1}<i_{2} \leq i_{n}}\left|A_{i_{1}} \cap A_{i_{2}}\right| \text { (número de elementos das intersecões dois a dois) }
$$

$\sum_{1 \leq i_{1}<i_{2}<i_{3} \leq i_{n}}\left|A_{i_{1}} \cap A_{i_{2}} \cap A_{i_{3}}\right|$ (número de elementos das interseções três a três) e assim por diante.

Exemplo 1. Um exame, composto por três questões, foi aplicado a uma turma de 25 alunos. Os resultados obtidos estão apresentados na tabela a seguir:

\begin{tabular}{|c|c|c|c|c|c|c|c|}
\hline Questão & 1 & 2 & 3 & 1 e 2 & 1 e 3 & 2 e 3 & 1,2 e 3 \\
\hline Acertos & 6 & 10 & 15 & 3 & 3 & 5 & 2 \\
\hline
\end{tabular}

Quantos alunos erraram as três questões?

Sejam: 
$A$ : o conjunto dos alunos que acertaram a primeira questão;

$B:$ o conjunto dos alunos que acertaram a segunda questão;

$C:$ o conjunto dos alunos que acertaram a terceira questão;

Pela tabela de dados e pelo principio de inclusão e exclusão, temos

$$
\begin{aligned}
|A \cup B \cup C|= & |A|+|B|+|C|-|A \cap B|-|A \cap C|-|B \cap C|+|A \cap B \cap C| \\
& =6+10+15-3-3-5+2=22
\end{aligned}
$$

$\log 0$

$$
\left|(A \cup B \cup C)^{C}\right|=25-22=3 \text { alunos }
$$

Exemplo 2. Quantos são os inteiros compreendidos entre 3001 e 7001 que são divisíveis por 3 ou 7 ?

Temos que determinar $|A \cup B|$ em que $A$ e $B$ são, respectivamente, os conjuntos dos múltiplos de 3 e 7 compreendidos entre 3001 e 7001.

\begin{tabular}{|cccc|}
\hline 3003 & 3006 & $\cdots$ & 6999 \\
$\square$ & $\curvearrowleft$ & & $\bigcap$ \\
$3 \times 1001$ & $3 \times 1002$ & $\ldots$ & $3 \times 2333$ \\
\hline
\end{tabular}

Assim $|A|=1333$

\begin{tabular}{|cccc|}
\hline 3003 & 3010 & $\cdots$ & 7000 \\
$\&$ & $\wp$ & & $\Omega$ \\
$7 \times 429$ & $7 \times 430$ & $\ldots$ & $7 \times 1000$ \\
\hline
\end{tabular}

Assim $|B|=572$

Resta saber quantos são os múltiplos de 3 e 7, ou seja, múltiplos de 21 compreendidos entre 3001 e 7001.

\begin{tabular}{|cccc|}
\hline 3003 & 3024 & $\cdots$ & 6993 \\
$\Downarrow$ & $\curvearrowleft$ & & $\curvearrowleft$ \\
$21 \times 143$ & $21 \times 144$ & $\ldots$ & $21 \times 333$ \\
\hline
\end{tabular}


Assim $|A \cap B|=191$

$\operatorname{Logo}|A \cup B|=1333+572-191=1714$. Há 1714 números que satisfazem o problema.

\subsection{FUNÇÃO TOTIENTE DE EULER E O PRINCÍPIO DE INCLUSÃO-EXCLUSÃO}

A função $\phi$ de Euler é tal que, para cada $n \in \mathbb{N}=\{1,2,3, \ldots\}, \phi(n)$ é a quantidade de números $k \in\{1,2,3, \ldots\}$ com a propriedade: $k$ e $n$ são relativamente primos, isto é, $k$ e $n$ não admitem primos em comum nas suas decomposições em fatores primos.

A fórmula $\phi(n)=n\left(1-\frac{1}{p_{1}}\right) \ldots\left(1-\frac{1}{p_{s}}\right)$ quando $n=p_{1}^{e_{1}} p_{2}^{e_{2}} \ldots p_{s}^{e_{s}}$ e $p_{1}, p_{2}, \ldots, p_{s}$ são os número primos presentes na decomposição de $n$ em fatores primos é mais uma aplicação importante do teorema de inclusão-exclusão.

Pelo teorema fundamental da aritmética, tem se $\operatorname{mdc}(k, n)=1$, se e só se $p_{i} \nmid k$, para todo $i$ tal que $1 \leq i \leq s$. Dessa forma,

$$
\phi(n)=\mid\left\{k \in \mathbb{N}: k \leq n \text { e } p_{1} \nmid k, \ldots, p_{s} \nmid k\right\} \mid
$$

Consideremos também

$$
A_{i}=\left\{k \in \mathbb{N}: k \leq n, \quad p_{i} \mid k\right\}, 1 \leq i \leq s
$$

$\operatorname{Temos}\left|A_{i}\right|=\frac{n}{p_{i}}$

Para $r$ interseções destes conjuntos temos:

$$
\begin{gathered}
\left|A_{i_{1}} \cap \ldots \cap A_{i_{r}}\right|=\left|\left\{k \in \mathbb{N}: k \leq n, p_{i_{1}}\left|k, \ldots, p_{i_{r}}\right| k\right\}\right| \\
=\frac{n}{p_{i_{1}} \ldots p_{i_{r}}}
\end{gathered}
$$

Assim

$$
\phi(n)=n-\left|A_{1} \cup \ldots \cup A_{S}\right|
$$

pelo princípio de inclusão-exclusão: 


$$
\begin{gathered}
\phi(n)=n-\left(\frac{n}{p_{1}}+\cdots+\frac{n}{p_{s}}\right)+\left(\frac{n}{p_{1} p_{2}}+\cdots+\frac{n}{p_{1} p_{s}}+\frac{n}{p_{2} p_{3}}+\cdots\right)-\cdots+(-1)^{s} \frac{n}{p_{1} p_{2} \ldots p_{s}} \\
=n\left[1-\left(\frac{1}{p_{1}}+\cdots+\frac{1}{p_{s}}\right)+\left(\frac{1}{p_{1} p_{2}}+\cdots+\frac{1}{p_{1} p_{s}}+\frac{1}{p_{2} p_{3}}+\cdots\right)-\cdots+(-1)^{s} \frac{1}{p_{1} p_{2} \ldots p_{s}}\right] \\
=n\left(1-\frac{1}{p_{1}}\right)\left(1-\frac{1}{p_{2}}\right) \cdots\left(1-\frac{1}{p_{s}}\right)
\end{gathered}
$$

\section{Exemplo 3.}

Calcular $\phi(n)$ para $n=2^{3} \cdot 3^{2} \cdot 5$.

Pela fórmula:

$$
\phi(360)=360\left(1-\frac{1}{2}\right)\left(1-\frac{1}{3}\right)\left(1-\frac{1}{5}\right)=96
$$

Pelo princípio de inclusão-exclusão:

$$
A_{1}=\{\text { mútiplos de } 2\} \quad A_{2}=\{\text { mútiplos de } 3\} \quad A_{3}=\{\text { mútiplos de } 5\}
$$

$A_{1} \cap A_{2}=\{$ múltiplos de 6$\} \quad A_{1} \cap A_{3}=\{$ mútiplos de 10$\} \quad A_{2} \cap A_{3}=\{$ mútiplos de 15$\}$

$$
A_{1} \cap A_{2} \cap A_{3}=\{\text { mútiplos de } 30\}
$$

$$
\phi(360)=360-\left(\frac{360}{2}+\frac{360}{3}+\frac{360}{5}\right)+\left(\frac{360}{6}+\frac{360}{10}+\frac{360}{15}\right)-\left(\frac{360}{30}\right)=96
$$




\section{TRIÂNGULO DE PASCAL}

O triângulo de Pascal, também conhecido como triângulo de Tartaglia, é um triângulo aritmético formado por números binomiais. Descoberto pelo matemático chinês Yang Hui (1238-1298) e estudado por Blaise Pascal (1623-1662).

A formação de triângulo de Pascal:

1

$1 \quad 1$

$\begin{array}{lll}1 & 2 & 1\end{array}$

$\begin{array}{llll}1 & 3 & 3 & 1\end{array}$

$\begin{array}{lllll}1 & 4 & 6 & 4 & 1\end{array}$

1

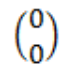

$\left(\begin{array}{l}1 \\ 0\end{array}\right) \quad\left(\begin{array}{l}1 \\ 1\end{array}\right)$

$\left(\begin{array}{l}2 \\ 0\end{array}\right) \quad\left(\begin{array}{l}2 \\ 1\end{array}\right) \quad\left(\begin{array}{l}2 \\ 2\end{array}\right)$

$\left(\begin{array}{lll}3 \\ 0\end{array}\right) \quad\left(\begin{array}{l}3 \\ 1\end{array}\right) \quad\left(\begin{array}{l}3 \\ 2\end{array}\right) \quad\left(\begin{array}{l}3 \\ 3\end{array}\right)$

$\left(\begin{array}{l}4 \\ 0\end{array}\right) \quad\left(\begin{array}{l}4 \\ 1\end{array}\right) \quad\left(\begin{array}{l}4 \\ 2\end{array}\right) \quad\left(\begin{array}{l}4 \\ 3\end{array}\right) \quad\left(\begin{array}{l}4 \\ 4\end{array}\right)$

$\left(\begin{array}{l}n \\ 0\end{array}\right) \quad \ldots$. $\left(\begin{array}{l}n \\ n\end{array}\right)$

Sendo $n$ e $p$, respectivamente, linha e coluna do elemento no triângulo, se contados a partir do zero, e:

$$
C_{n}^{p}=\left(\begin{array}{l}
n \\
p
\end{array}\right)=\frac{n !}{p !(n-p) !}
$$

\subsection{RELAÇÃO DE STIFEL}

Essa propriedade permite a construção rápida do triângulo, nessa relação temos que:

$\left(\begin{array}{l}n \\ p\end{array}\right)=\left(\begin{array}{l}n-1 \\ p-1\end{array}\right)+\left(\begin{array}{c}n-1 \\ p\end{array}\right)$, ou seja, cada termo é obtido pela soma dos dois elementos imediatamente superiores na linha anterior.

1

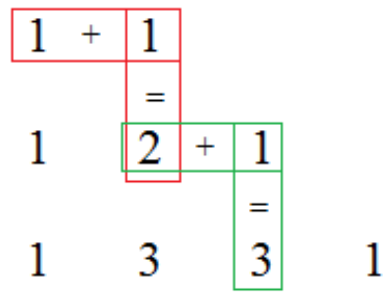

$\left(\begin{array}{l}0 \\ 0\end{array}\right)$

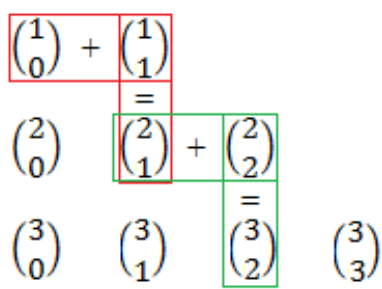


A demonstração da relação de Stifel é decorrente apenas da definição de número binomial e manipulações algébricas.

$$
\begin{gathered}
\left(\begin{array}{c}
n-1 \\
p-1
\end{array}\right)+\left(\begin{array}{c}
n-1 \\
p
\end{array}\right)=\frac{(n-1) !}{(p-1) !(n-p) !}+\frac{(n-1) !}{p !(n-p-1) !} \\
\quad=\frac{p(n-1) !}{p(p-1) !(n-p) !}+\frac{(n-p)(n-1) !}{p !(n-p)(n-p-1) !}=\frac{(p+(n-p))(n-1) !}{(n-p) ! p !} \\
=\frac{n(n-1) !}{(n-p) ! p !}=\left(\begin{array}{l}
n \\
p
\end{array}\right)
\end{gathered}
$$

Se olharmos para uma das linhas do triângulo, podemos perceber que há simetria entre seus elementos, isso se deve ao fato de que na linha $n$, começada por $C_{n}^{0}$ e terminada por $C_{n}^{n}$ se deslocarmos $p$ unidades a partir do inicio $\left(C_{n}^{p}\right)$ e voltarmos $\mathrm{p}$ unidades a partir do fim $\left(C_{n}^{n-p}\right)$ temos que $C_{n}^{p}=C_{n}^{n-p}$, esse números são iguais e são chamados de combinações complementares. Por exemplo, a complementar de $C_{5}^{2}$ é $C_{5}^{5-2}=C_{5}^{3}$. Em outras palavras, elementos de uma mesma linha, equidistantes dos extremos, são iguais.

Justificativa:

$$
\left(\begin{array}{c}
n \\
n-p
\end{array}\right)=\frac{n !}{(n-p) !(n-(n-p)) !}=\frac{n !}{(n-p) ! p !}=\left(\begin{array}{l}
n \\
p
\end{array}\right)
$$

\subsection{REGRA DA SOMA DE UMA LINHA}

Repare que a soma dos elementos de uma linha no triângulo de pascal é uma potência de 2.

$$
\begin{aligned}
& \text { ( linha } 0) \quad 1=1=2^{0} \\
& \text { (linha 1) } 1+1 \quad=2=2^{1} \\
& \text { ( linha 2) } 1+2+1=4=2^{2} \\
& \text { ( linha 3) } 1+3+3+1=8=2^{3} \\
& \text { (linha 4) } 1+4+6+4+1=16=2^{4}
\end{aligned}
$$

Assim temos que a soma dos elementos da $n$-ésima linha será $2^{n}$, ou seja:

$$
\left(\begin{array}{l}
n \\
0
\end{array}\right)+\left(\begin{array}{l}
n \\
1
\end{array}\right)+\left(\begin{array}{l}
n \\
2
\end{array}\right)+\cdots+\left(\begin{array}{c}
n \\
n-1
\end{array}\right)+\left(\begin{array}{l}
n \\
n
\end{array}\right)=2^{n}
$$


Justificativa: Seja $\left(\begin{array}{l}n \\ p\end{array}\right)$ o número de subconjuntos do conjunto $A=\{1,2,3, \ldots, n\}$ com $p$ elementos. Logo, $\left(\begin{array}{l}n \\ 0\end{array}\right)+\left(\begin{array}{l}n \\ 1\end{array}\right)+\left(\begin{array}{l}n \\ 2\end{array}\right)+\cdots+\left(\begin{array}{c}n \\ n-1\end{array}\right)+\left(\begin{array}{l}n \\ n\end{array}\right)$ é o total de subconjuntos de $A$. No entanto para formar um subconjunto de $A$ devemos marcar cada elemento em $A$ com o símbolo $\epsilon$ (indicando que o elemento foi escolhido para o subconjunto) ou $\notin$ (indicando que o elemento não foi escolhido para o subconjunto). Sendo assim, como para cada elemento há duas possibilidades $(\in$, ou $\notin)$, temos $2 \times 2 \times \ldots \times 2=2^{n}$.

\subsection{REGRA DA SOMA DE UMA COLUNA}

A soma dos elementos de uma coluna, a partir do primeiro, é igual ao elemento que se encontra avançado uma linha e uma coluna em relação à última parcela da adição.

$$
\left(\begin{array}{l}
p \\
p
\end{array}\right)+\left(\begin{array}{c}
p+1 \\
p
\end{array}\right)+\left(\begin{array}{c}
p+2 \\
p
\end{array}\right)+\cdots+\left(\begin{array}{c}
p+n \\
p
\end{array}\right)=\left(\begin{array}{c}
p+n+1 \\
p+1
\end{array}\right)
$$

Veja o esquema.

1

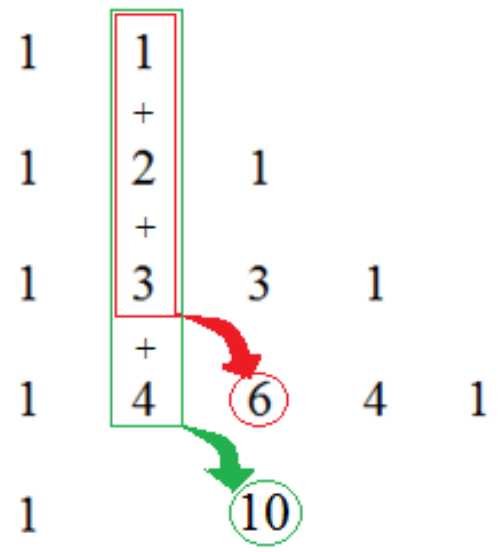

Justificativa:

Coluna $p+1$ : $\left(\begin{array}{l}0 \\ 0\end{array}\right)$

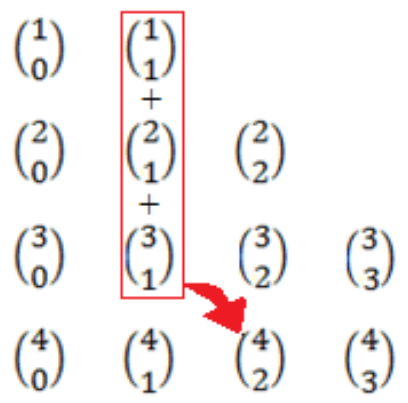

$\left(\begin{array}{l}4 \\ 4\end{array}\right)$ 


\begin{tabular}{|c|l|}
\hline $\left.\begin{array}{l}p+1 \\
p+1\end{array}\right)=\left(\begin{array}{c}p \\
p+1\end{array}\right)+\left(\begin{array}{l}p \\
p\end{array}\right)$ & $\begin{array}{l}\text { Coma soma telescópica } \\
\text { simplificando parcelas iguais que } \\
\left(\begin{array}{c}p+2 \\
p+1\end{array}\right)=\left(\begin{array}{c}p+1 \\
p+1\end{array}\right)+\left(\begin{array}{c}p+1 \\
p\end{array}\right) \\
\text { aparecem em membros opostos, } \\
\left(\begin{array}{c}p+3 \\
p+1\end{array}\right)=\left(\begin{array}{c}p+2 \\
p+1\end{array}\right)+\left(\begin{array}{c}p+2 \\
p\end{array}\right) \\
\text { obtém-se o desejado. } \\
\left(\begin{array}{c}p+n \\
p+1\end{array}\right)=\left(\begin{array}{c}p+n-1 \\
p+1\end{array}\right)+\left(\begin{array}{c}p+n-1 \\
p\end{array}\right) \\
\left(\begin{array}{c}p+n+1 \\
p+1\end{array}\right)=\left(\begin{array}{c}p+n \\
p+1\end{array}\right)+\left(\begin{array}{c}p+n \\
p\end{array}\right)\end{array}$ \\
\hline
\end{tabular}

Tendo em vista que $\left(\begin{array}{c}p \\ p+1\end{array}\right)=0$, temos:

$$
\left(\begin{array}{c}
p+n+1 \\
p+1
\end{array}\right)=\left(\begin{array}{c}
p \\
p
\end{array}\right)+\left(\begin{array}{c}
p+1 \\
p
\end{array}\right)+\left(\begin{array}{c}
p+2 \\
p
\end{array}\right)+\cdots+\left(\begin{array}{c}
p+n \\
p
\end{array}\right)
$$

\subsection{REGRA DA SOMA DE UMA DIAGONAL}

A soma dos elementos de uma diagonal, começando do primeiro, é igual ao elemento que se encontra imediatamente abaixo da última parcela.

$$
\left(\begin{array}{l}
n \\
0
\end{array}\right)+\left(\begin{array}{c}
n+1 \\
1
\end{array}\right)+\left(\begin{array}{c}
n+2 \\
2
\end{array}\right)+\cdots+\left(\begin{array}{c}
n+p \\
p
\end{array}\right)=\left(\begin{array}{c}
n+p+1 \\
p
\end{array}\right)
$$

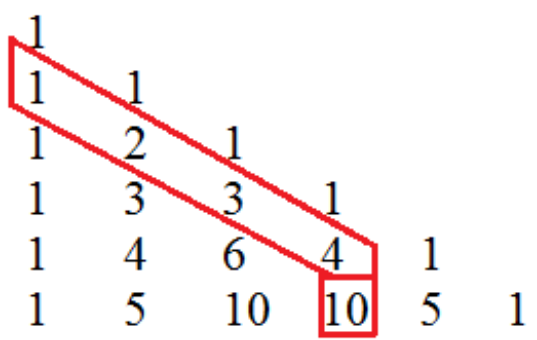

Justificativa:

Com sucessivas combinações complementares e a regra da soma de uma coluna, obtemos:

$$
\begin{aligned}
\left(\begin{array}{c}
n \\
0
\end{array}\right)+\left(\begin{array}{c}
n+1 \\
1
\end{array}\right) & +\left(\begin{array}{c}
n+2 \\
2
\end{array}\right)+\cdots+\left(\begin{array}{c}
n+p \\
p
\end{array}\right)=\left(\begin{array}{c}
n \\
n
\end{array}\right)+\left(\begin{array}{c}
n+1 \\
n
\end{array}\right)+\left(\begin{array}{c}
n+2 \\
n
\end{array}\right)+\cdots+\left(\begin{array}{c}
n+p \\
n
\end{array}\right) \\
= & \left(\begin{array}{c}
n+p+1 \\
n+1
\end{array}\right)=\left(\begin{array}{c}
n+p+1 \\
p
\end{array}\right)
\end{aligned}
$$




\subsection{BINÔMIO DE NEWTON}

Teorema 2. Para todo, $a, b \in R$ e $n \in N$ vale que

$(a+b)^{n}=\left(\begin{array}{l}n \\ 0\end{array}\right) a^{n}+\left(\begin{array}{l}n \\ 1\end{array}\right) a^{n-1} b+\left(\begin{array}{l}n \\ 2\end{array}\right) a^{n-2} b^{2}+\cdots+\left(\begin{array}{c}n \\ n-1\end{array}\right) a b^{n-1}+\left(\begin{array}{l}n \\ n\end{array}\right) b^{n}$

Prova por indução.

Para $n=1$, temos $(a+b)^{1}=\left(\begin{array}{l}1 \\ 0\end{array}\right) a^{1}+\left(\begin{array}{l}1 \\ 1\end{array}\right) a^{1-1} b=a+b$, portanto a fórmula é valida para $n=1$.

Suponhamos agora, por hipótese de indução, que a fórmula seja válida para $n=k \geq$ 1 , isto é,

$$
(a+b)^{k}=\left(\begin{array}{l}
k \\
0
\end{array}\right) a^{k}+\left(\begin{array}{l}
k \\
1
\end{array}\right) a^{k-1} b+\left(\begin{array}{l}
k \\
2
\end{array}\right) a^{k-2} b^{2}+\cdots+\left(\begin{array}{c}
k \\
k-1
\end{array}\right) a b^{k-1}+\left(\begin{array}{l}
k \\
k
\end{array}\right) b^{k}
$$

Segue que $(a+b)^{k+1}=(a+b)^{k} \cdot(a+b)=(a+b)^{k} \cdot a+(a+b)^{k} \cdot b=$

$$
\begin{gathered}
=\left(\begin{array}{l}
k \\
0
\end{array}\right) a^{k+1}+\left(\begin{array}{c}
k \\
1
\end{array}\right) a^{k} b+\left(\begin{array}{l}
k \\
2
\end{array}\right) a^{k-1} b^{2}+\cdots+\left(\begin{array}{c}
k \\
k-1
\end{array}\right) a^{2} b^{k-1}+\left(\begin{array}{l}
k \\
k
\end{array}\right) a b^{k}+\left(\begin{array}{l}
k \\
0
\end{array}\right) a^{k} b \\
+\left(\begin{array}{c}
k \\
1
\end{array}\right) a^{k-1} b^{2}+\left(\begin{array}{l}
k \\
2
\end{array}\right) a^{k-2} b^{3}+\cdots+\left(\begin{array}{c}
k \\
k-1
\end{array}\right) a b^{k}+\left(\begin{array}{l}
k \\
k
\end{array}\right) b^{k+1}
\end{gathered}
$$

Usando a relação de Stifel, obtemos

$$
\begin{aligned}
(a+b)^{k+1}= & \left(\begin{array}{c}
k+1 \\
0
\end{array}\right) a^{k+1}+\left(\begin{array}{c}
k+1 \\
1
\end{array}\right) a^{k} b+\left(\begin{array}{c}
k+1 \\
2
\end{array}\right) a^{k-1} b^{2}+\cdots+\left(\begin{array}{c}
k+1 \\
k
\end{array}\right) a b^{k} \\
& +\left(\begin{array}{c}
k+1 \\
k+1
\end{array}\right) b^{k+1}
\end{aligned}
$$

Portanto, a fórmula é válida para $k+1$, logo é válida para todo $n \in N$.

O Binômio de Newton é uma importante ferramenta na solução de problemas da genética.

Exemplo 4. A cor da pele humana é determinada por, no mínimo, dois pares de genes alelos.

- $B-b$

- $\quad N-n$

Em que, 
$N$ e $B$ : determinam maior quantidade de melanina (alelos efetivos).

$n$ e $b$ : determinam menor quantidade de melanina (alelos não efetivos).

Assim, pessoas $N N B B$ serão negras, $n n b b$ serão brancas e $N N b b$ serão mulatos médios. Os cruzamentos possíveis entre um casal de indivíduos, mulatos médios, pode ser representado pelo esquema abaixo.
Geração
$N n B b$
$\times$
$\mathrm{NnBb}$
Gametas
$\begin{array}{llllllll}N B & N b & n B & n b & \times & N B & N b & n B\end{array}$

\begin{tabular}{|c|c|c|c|c|}
\hline$\times$ & $\boldsymbol{N B}$ & $\boldsymbol{N b}$ & $\boldsymbol{n B}$ & $\boldsymbol{n b}$ \\
\hline \multirow{2}{*}{$\boldsymbol{B}$} & $N N B B$ & $N N B b$ & $N n B B$ & $N n B b$ \\
& Negro & Mulato escuro & Mulato escuro & Mulato médio \\
\hline \multirow{2}{*}{$\boldsymbol{N b}$} & $N N B b$ & $N N b b$ & $N n B b$ & $N n b b$ \\
& Mulato escuro & Mulato médio & Mulato médio & Mulato claro \\
\hline \multirow{n}{*}{$\boldsymbol{B}$} & $N n B B$ & $N n B b$ & $n n B B$ & $n n B b$ \\
& Mulato escuro & Mulato médio & Mulato médio & Mulato claro \\
& $N n B b$ & $N n b b$ & $n n B b$ & $n n b b$ \\
& Mulato médio & Mulato claro & Mulato claro & Branco \\
\hline
\end{tabular}

Tabela de proporção

\begin{tabular}{|c|c|c|}
\hline Fenótipos & Genótipos & Proporção fenotípica \\
\hline Negro & $N N B B$ & $\frac{\mathbf{1}}{16}$ \\
\hline Mulato escuro & $N N B b$ ou $N n B B$ & $\frac{\mathbf{4}}{16}$ \\
\hline Mulato médio & $N n B b, N N b b$ ou & $\frac{\mathbf{6}}{16}$ \\
\hline Mulato claro & $n n B B$ & $\frac{\mathbf{4}}{16}$ \\
\hline Branco & $N n b b$ ou $n n B b$ & $\frac{\mathbf{1}}{16}$ \\
\hline
\end{tabular}


Os números na distribuição fenotípica $(1,4,6,4,1)$ correspondem à $4^{\mathrm{a}}$ linha do triângulo de Pascal. Se denotarmos por $(a)$ os alelos efetivos $(N$ e $B)$, por $(c)$ os alelos não efetivos $(n$ e $b)$ e desenvolver o binômio $(a+c)^{n}, \operatorname{com} n=4$ temos:

$$
(a+c)^{4}=1 a^{4}+4 a^{3} c+6 a^{2} c^{2}+4 a c^{3}+1 c^{4}
$$

Os expoentes podem ser interpretados da seguinte maneira: $a^{4}$ significa a presença de 4 alelos efetivos (indivíduo negro), $a^{3} c$ a presença de 3 alelos efetivos e 1 alelo não efetivo (mulato escuro), e assim por diante. Além disso, o total de cruzamentos possíveis pode ser obtido pela regra da soma de uma linha, mais precisamente a linha 4, em que o resultado é $2^{4}=16$.

Exemplo 5. Um sitiante possui uma coelha que está prenha e que terá seis filhotes. Qual a probabilidade de ela ter três casais, sem importar a ordem?

Solução:

Considerando que a probabilidade de ter macho ou fêmea seja igual a 50\%, e desenvolvendo o binômio $(m+f)^{n}$, com $n=6$, o que devemos fazer é procurar o termo $m^{3} f^{3}$.

$$
(m+f)^{6}=m^{6}+6 m^{5} f+15 m^{4} f^{2}+\mathbf{2 0 m ^ { 3 }} \boldsymbol{f}^{3}+15 m^{2} f^{4}+6 m f^{5}+f^{6}
$$

Dessa forma, a probabilidade de obter três casais é

$$
20 m^{3} f^{3}=20 \cdot\left(\frac{1}{2}\right)^{3} \cdot\left(\frac{1}{2}\right)^{3}=\frac{5}{16}=0,3125=31,25 \%
$$

\subsection{SEQUÊNCIA DE FIBONACCI E O TRIÂNGULO DE PASCAL}

A sequência de Fibonacci, $\{1,1,2,3,5,8,13,21,34, \ldots\}$, em que $F_{1}=F_{2}=1$ e $F_{n}=F_{n-1}+F_{n-2}, \quad \forall n \geq 3$, pode ser definida a partir do triângulo de Pascal. A soma dos números das diagonais é um número de Fibonacci. Veja o esquema a seguir. 


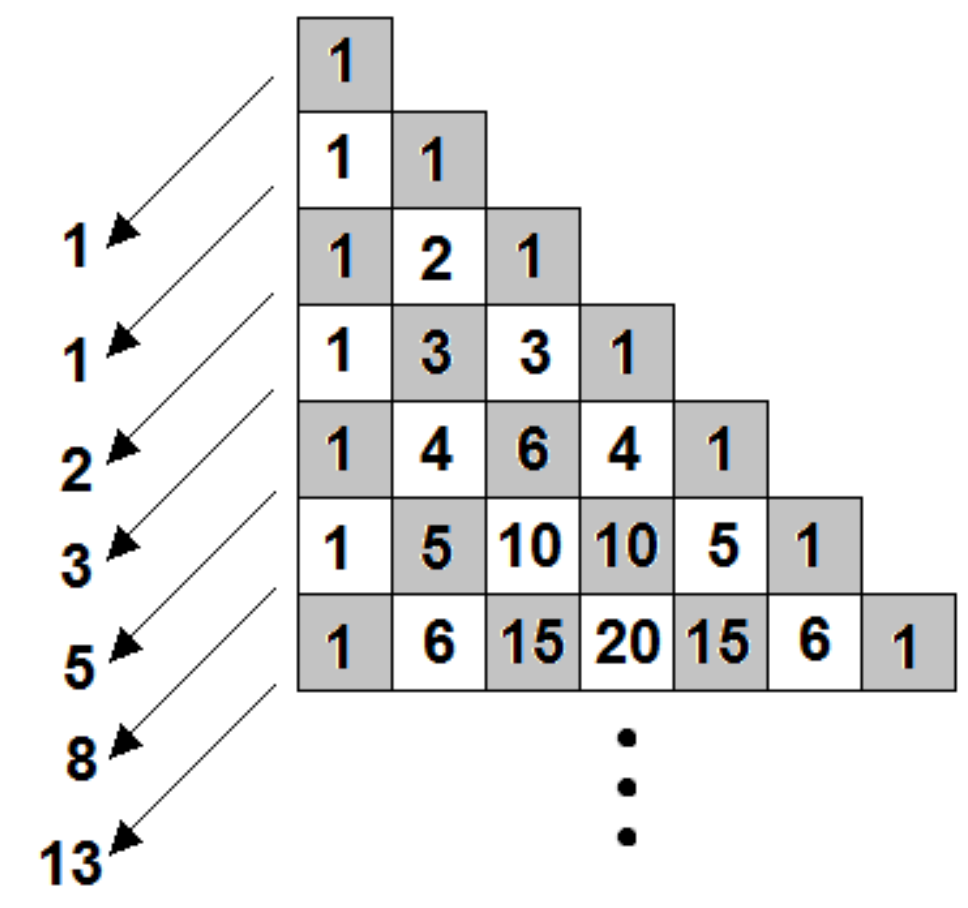

Justificativa:

Se olharmos para três diagonais quaisquer que sejam consecutivas, percebermos que é decorrente da relação de Stifel, pois cada termo é obtido a partir da soma do número que está acima com o número à sua direita.

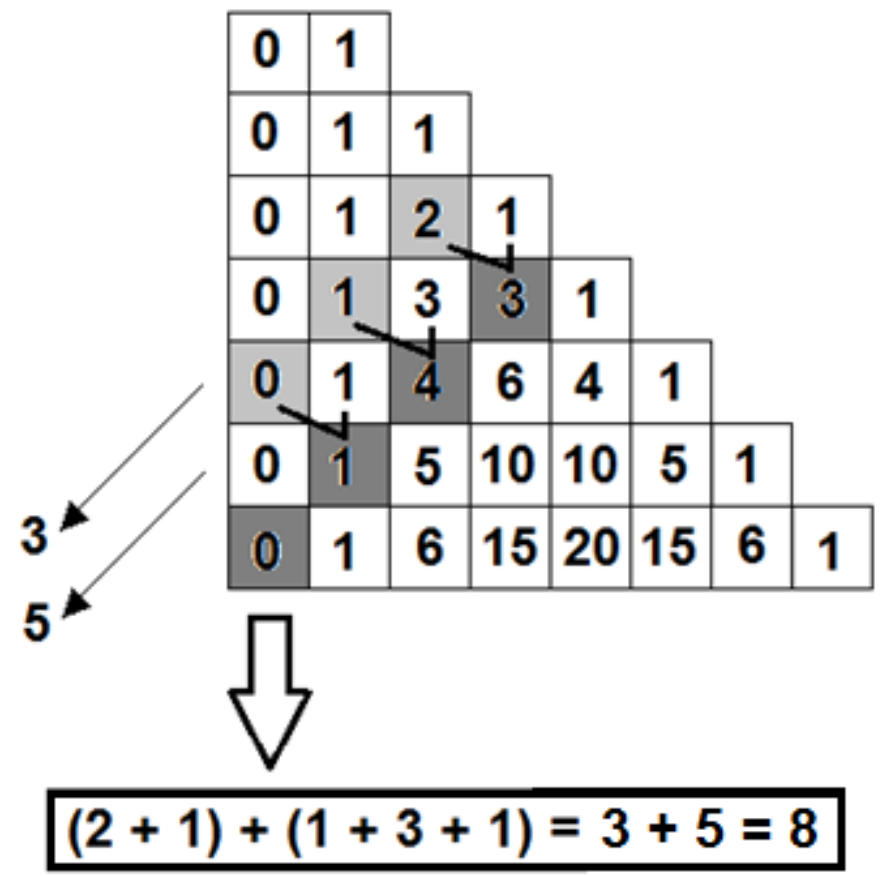




\section{RELAÇÃO BINÁRIA}

A relação binária ou 2-ária é uma relação que forma um conjunto de pares ordenados.

\section{Definição}

Uma relação binária de $A$ em $B$ é definida como sendo um subconjunto do produto cartesiano entre o conjunto A e o conjunto B. Isto é, uma relação R é um conjunto de pares ordenados. Um subconjunto de $A \times A$ pode ser chamada de relação binária sobre A.

Em outras palavras, uma relação binária $\mathbf{R}$ sobre dois universos $\mathrm{A}$ e $\mathrm{B}$ pode ser representada simbolicamente por:

$$
R \subseteq A \times B
$$

O número de relações binárias $\mathbf{R}$ definidas no conjunto $X=\{1,2,3, \ldots, n\}$ é igual ao número de matrizes quadradas de ordem $\mathbf{n}$ devido à correspondência biunívoca canônica entre relações binárias $\mathbf{R}$ sobre $\mathrm{X}$ e matrizes quadradas de ordem $\mathrm{n}$ cujos elementos pertencem a $\{0,1\}$. O número de relações binárias é $2^{n^{2}}$.

\section{Exemplo 6.}

$$
\begin{gathered}
X=\{1,2,3\} \\
R=\{(1,2),(1,3),(2,2),(2,3)\} \\
M_{R}=\left(\begin{array}{lll}
r_{1,1} & r_{1,2} & r_{1,3} \\
r_{2,1} & r_{2,2} & r_{2,3} \\
r_{3,1} & r_{3,2} & r_{3,3}
\end{array}\right) \Rightarrow M_{R}=\left(\begin{array}{lll}
0 & 1 & 1 \\
0 & 1 & 1 \\
0 & 0 & 0
\end{array}\right)
\end{gathered}
$$

Nesse caso, é possível construir $2^{3^{2}}$ relações binárias com os elementos de $X$.

\subsection{TIPOS DE RELAÇÕES BINÁRIAS}

Dada uma relação binária $R \subseteq A \times B$, podemos classificá-la como:

- total

$$
(\forall a \in A)(\exists b \in B), a R b
$$

Ou seja, todo elemento de A se relaciona com algum elemento de B. 
- sobrejetora

$$
(\forall b \in B)(\exists a \in A), a R b
$$

É o inverso da total, todo elemento de B é relacionado com algum elemento de A.

- funcional

$$
(\forall a \in A)\left(\forall b_{1}, b_{2} \in B\right), a R b_{1} \wedge a R b_{2} \rightarrow\left(b_{1}=b_{2}\right)
$$

Ou seja, um elemento de A não pode se relacionar com mais de um elemento de B.

- injetora

$$
\left(\forall a_{1}, a_{2} \in A\right)(\forall b \in B)\left(\forall b_{1}, b_{2} \in B\right), a_{1} R b \wedge a_{2} R b \rightarrow\left(a_{1}=a_{2}\right)
$$

Um elemento de B não pode se relacionar com dois ou mais elementos distintos de A.

\subsection{PROPRIEDADE DAS RELAÇÕES}

Considere as seguintes relações sobre um conjunto $X=\{1,2,3\}$

$$
\begin{gathered}
R_{1}=\{(1,1),(1,2),(1,3),(2,1),(2,3),(3,1),(3,2),(3,3)\} \\
R_{2}=\{(1,1),(1,2),(2,1),(2,2),(3,3)\} \\
R_{3}=\{(1,3),(2,1)\} \\
R_{4}=\emptyset
\end{gathered}
$$

$R_{5}=X^{2}$, a relação universal.

\subsubsection{RELAÇÃO REFLEXIVA}

Uma relação $\mathbf{R}$ é classificada como reflexiva se todos os elementos se relacionam com si próprios. Ou seja, $x R x$ para todo $x \in X$, isto é, se $(x, x) \in R$ para todo $x \in X$.

$$
(\forall x \in X)((x, x) \in R)
$$

Dos exemplos citados apenas $R_{2}$ e $R_{5}$ são reflexivas.

Uma relação é irreflexiva se nenhum elemento se relacionar com si próprio.

$$
(\forall x \in X)((x, x) \notin R)
$$


Em com conjunto finito com $\mathbf{n}$ elementos existem $2^{n^{2}}$ relações, das quais, reflexivas ou irreflexivas, são $2^{n^{2}-n}$.

\subsubsection{RELAÇÃO SIMÉTRICA}

Uma relação é simétrica se para qualquer $x$ que se relacione com $y$ implicar $y$ ser relacionado $\operatorname{com} x$.

Exemplo: A relação " $a$ é irmão de $b$ " é simétrica, pois, com isso, " $b$ é irmão de $a$ ". Formalmente, uma relação binária é simétrica se para qualquer $a R b \rightarrow b R a$.

$$
(\forall x, y \in X)((x, y) \in R \Rightarrow(y, x) \in R)
$$

Para um conjunto finito com $\mathbf{n}$ elementos, há $2^{\frac{n^{2}+n}{2}}$ relações binárias simétricas. As relações $R_{1}, R_{2}$ e $R_{5}$ são simétricas.

Uma relação é antissimétrica se $x$ relaciona com $y$ implica $y$ não se relacionando com $x$. As relações $R_{3}$ e $R_{4}$ são antissimétricas.

$$
(\forall x, y \in X)((x, y) \in R \Rightarrow(y, x) \notin R)
$$

\subsection{NÚMERO DE FUNÇÕES}

O número de funções $f$, cujo domínio de função é $D(f)=X=\{1,2,3, \ldots, n\}$ e cujo conjunto de valores $R(f)$ está contida no conjunto $Y=\{1,2, \ldots, m\}$ é $m^{n}$ porque as possibilidades para $f(1), f(2), \ldots, f(n)$ são todas iguais a $m$, pois:

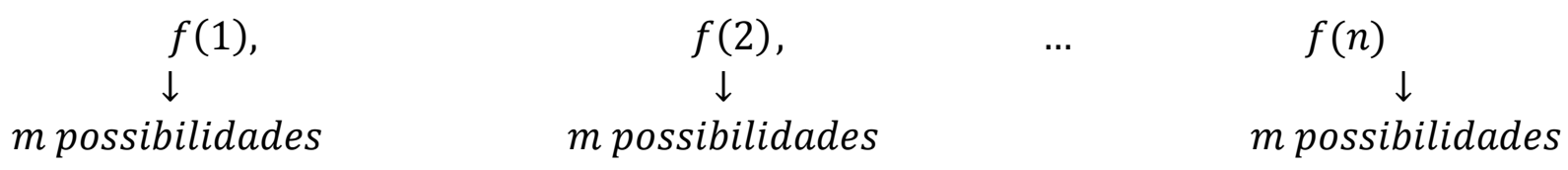

pelo princípio fundamental de contagem temos que existem $m \cdot m \cdot m \cdots m=m^{n}$ funções. 


\subsubsection{NÚMERO DE FUNÇÕES INJETORAS}

Dados $X=\{1,2,3, \ldots, n\}, Y=\{1,2, \ldots, m\}$ e naturalmente $n \leq m$, temos que número de funções injetoras $f: X \rightarrow Y$ é dado por $m^{\underline{n}}=\frac{m !}{(m-n) !}$, pois nesse caso $f(1), f(2), \ldots, f(n)$ devem assumir valores distinto.

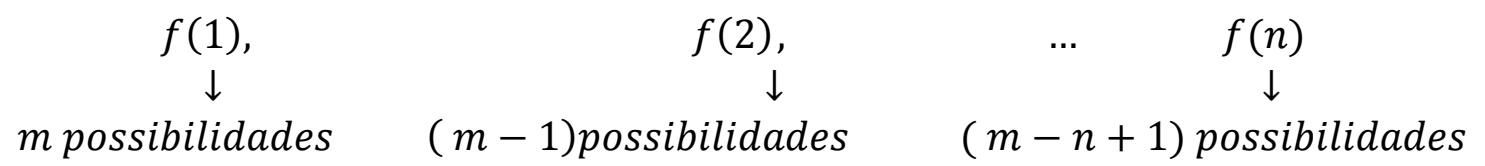

Portanto, pelo princípio fundamental de contagem há $m \cdot(m-1) \cdots(m-n+1)=$ $m^{\underline{n}}$ funções injetoras $f: X \rightarrow Y$.

\subsubsection{NÚMERO DE FUNÇÕES BIJETORAS}

Dados $X=\{1,2,3, \ldots, n\}, Y=\{1,2, \ldots, m\}$ e $n=m$ temos $m$ ! bijeções de $X$ em $Y$, pois trata-se de um caso particular da situação anterior, dessa forma temos:

$\begin{array}{cccc}f(1), & f(2), & \ldots & f(n) \\ \downarrow & \downarrow & \downarrow \\ \text { mpossibilidades } & (m-1) \text { possibilidades } & & 1 \text { possibilidade }\end{array}$

\subsubsection{NÚMERO DE FUNÇÕES SOBREJETORAS}

Dados $X=\{1,2,3, \ldots, n\}, Y=\{1,2, \ldots, m\}$ e $n \geq m$ o número de funções sobrejetoras $f: X \rightarrow Y$ é dado por:

$$
\left(\begin{array}{c}
m \\
m
\end{array}\right) m^{n}-\left(\begin{array}{c}
m \\
m-1
\end{array}\right)(m-1)^{n}+\cdots+(-1)^{m-1}\left(\begin{array}{c}
m \\
1
\end{array}\right) 1^{n}=\sum_{k=0}^{m-1}(-1)^{k} \cdot\left(\begin{array}{c}
m \\
m-k
\end{array}\right) \cdot(m-k)^{n}
$$

Em destaque, temos o caso particular para compreender a estruturação.

Número de funções sobrejetoras $f: X=\{1,2,3,4,5\}, Y=\{1,2,3\}$

$$
\begin{array}{cc}
U=\{f: X \rightarrow Y, \quad X=\{1,2,3,4,5\} & Y=\{1,2,3\}\} \\
A_{1}=\{f \in U: 1 \notin Y\} & A_{2}=\{f \in U: 2 \notin Y\}
\end{array}
$$


Note que $|U|=3^{5},\left|A_{1}\right|=\left|A_{2}\right|=\left|A_{3}\right|=2^{5},\left|A_{1} \cap A_{2}\right|=\left|A_{1} \cap A_{3}\right|=\left|A_{2} \cap A_{3}\right|=$ $1^{5}$, e $\left|A_{1} \cap A_{2} \cap A_{3}\right|=0^{5}$.

Pelo princípio de inclusão e exclusão segue a solução

$$
\left(\begin{array}{l}
3 \\
3
\end{array}\right) 3^{5}-\left(\begin{array}{l}
3 \\
2
\end{array}\right) 2^{5}+\left(\begin{array}{l}
3 \\
1
\end{array}\right) 1^{5}-\left(\begin{array}{l}
3 \\
0
\end{array}\right) 0^{5}=150 \text { funções sobrejetoras }
$$

\section{Exemplo 7.}

O cartão nacional de saúde (CNS) possui 15 dígitos que são escolhidos do conjunto $\{0,1,2, \ldots, 9\}$. Quantos são os CNS que apresentam todos os dígitos?

Solução:

$$
\left(\begin{array}{l}
9 \\
9
\end{array}\right) 9^{15}-\left(\begin{array}{l}
9 \\
8
\end{array}\right) 8^{15}+\left(\begin{array}{l}
9 \\
7
\end{array}\right) 7^{15}-\left(\begin{array}{l}
9 \\
6
\end{array}\right) 6^{15}+\left(\begin{array}{l}
9 \\
5
\end{array}\right) 5^{15}-\left(\begin{array}{l}
9 \\
4
\end{array}\right) 4^{15}+\left(\begin{array}{l}
9 \\
3
\end{array}\right) 3^{15}-\left(\begin{array}{l}
9 \\
2
\end{array}\right) 2^{15}+\left(\begin{array}{l}
9 \\
1
\end{array}\right) 1^{15}
$$




\section{PERMUTAÇÃO}

Uma permutação de $n$ elementos ou uma $n$-permutação é uma função injetora $f$ (e, portanto sobrejetora) cujo domínio de definição $D(f)$ e cujo conjunto de valores $R(f)$ são $\operatorname{ambos} D(f)=R(f)=\{1,2, \ldots, n\}$.

O conjunto de todas as $n$-permutação é conhecido como o grupo de permutações $S_{n}$ e $\left|S_{n}\right|=n$ !

Por exemplo,

\begin{tabular}{|c|c|c|c|c|c|c|c|c|c|}
\hline$S_{n}$ & \multicolumn{6}{|c|}{ Representação } & \multicolumn{3}{|c|}{$\begin{array}{c}\text { Notação } \\
\text { sequencial } \\
\text { respectiva }\end{array}$} \\
\hline$S_{1}$ & \multicolumn{6}{|c|}{$1 \rightarrow 1$} & \multicolumn{3}{|c|}{1} \\
\hline$S_{2}$ & \multicolumn{3}{|c|}{$\begin{array}{l}1 \rightarrow 1 \\
2 \rightarrow 2\end{array}$} & \multicolumn{3}{|c|}{$\begin{array}{l}1 \rightarrow 2 \\
2 \rightarrow 1\end{array}$} & \multicolumn{2}{|c|}{12} & 21 \\
\hline$S_{3}$ & $\begin{array}{l}1 \rightarrow 1 \\
2 \rightarrow 2 \\
3 \rightarrow 3\end{array}$ & $\begin{array}{l}1 \rightarrow 2 \\
2 \rightarrow 3 \\
3 \rightarrow 1\end{array}$ & $\begin{array}{l}1 \rightarrow 3 \\
2 \rightarrow 1 \\
3 \rightarrow 2\end{array}$ & $\begin{array}{l}1 \rightarrow 1 \\
2 \rightarrow 3 \\
3 \rightarrow 2\end{array}$ & $\begin{array}{l}1 \rightarrow 3 \\
2 \rightarrow 2 \\
3 \rightarrow 1\end{array}$ & $\begin{array}{l}1 \rightarrow 2 \\
2 \rightarrow 1 \\
3 \rightarrow 3\end{array}$ & $\begin{array}{l}123 \\
132\end{array}$ & $\begin{array}{l}231 \\
321\end{array}$ & $\begin{array}{l}312 \\
213\end{array}$ \\
\hline
\end{tabular}

A notação sequencial para permutações permite a listagem das 4 - permutação a partir das 3 - permutação.

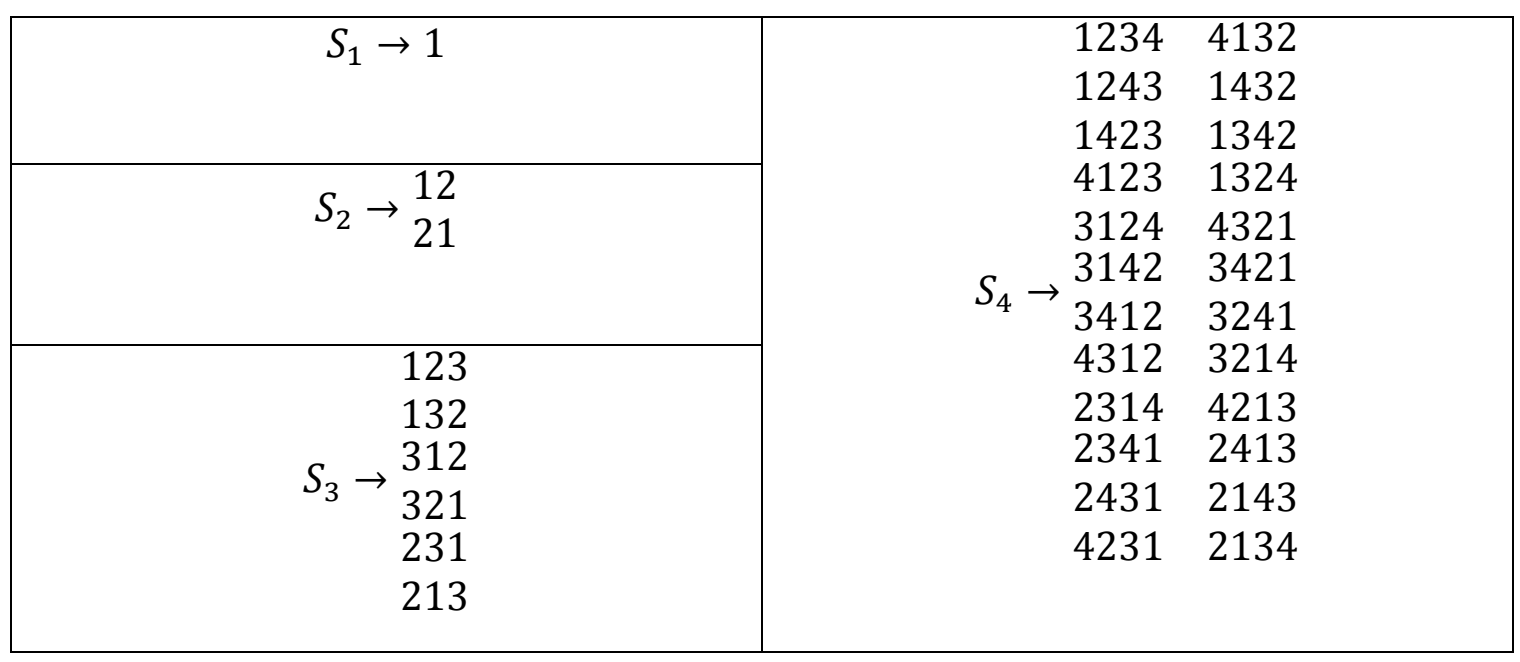




\subsection{NÚMERO DE PERMUTAÇÕES SUJEITA A RESTRIÇÕES}

O cálculo do número das $3-$ permutação sujeitas às restrições $f(1) \neq 2, f(2) \neq 3$, $f(3) \neq 1$ e $f(3) \neq 3$ é obtida através do quadrado:

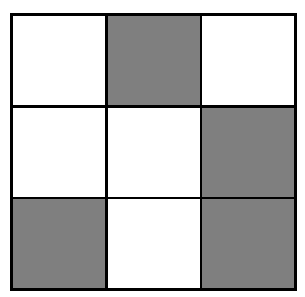

o qual é decomposto em
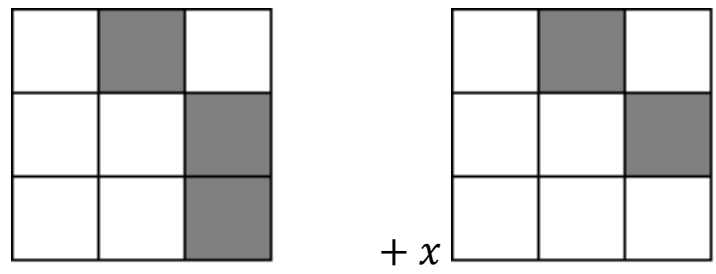

cujo polinômio-torre

$$
\left(1+3 x+2 x^{2}\right)+x\left(1+2 x+x^{2}\right)=1+4 x+4 x^{2}+x^{3}
$$

e o cálculo do número de 3 - permutação é obtido pela fórmula (por convenção $0 !=1$ )

$$
1 \cdot 3 !-4 \cdot 2 !+4 \cdot 1 !-1 \cdot 0 !=6-8+4-1=1
$$

Uma única permutação, a saber, 312 satisfaz as restrições, pois 312 significa

$$
\begin{aligned}
& f(1)=3 \\
& f(2)=1 \\
& f(3)=2
\end{aligned}
$$

A cada quadrado $4 \times 4 B$ corresponde um polinômio-torre de $4^{\circ}$ grau

$$
R(x, B)
$$

cujo coeficiente independente é 1 , e o coeficiente em $x$ é o número de colunas de uma peça sobre $n$ quadradinhos hachurados de $B$, cujo coeficiente em $x^{2}$ é o número de maneiras de 
colocação de duas peças que não se situem sobre a mesma linha e a mesma coluna de $B$, cujo coeficiente em $x^{3}$ é o número de colunas de três peças sobre $n$ quadradinhos hachurados de $B$, de modo que as três peças não estejam situadas sobre a mesma linha e a mesma coluna de $B$ e assim por diante.
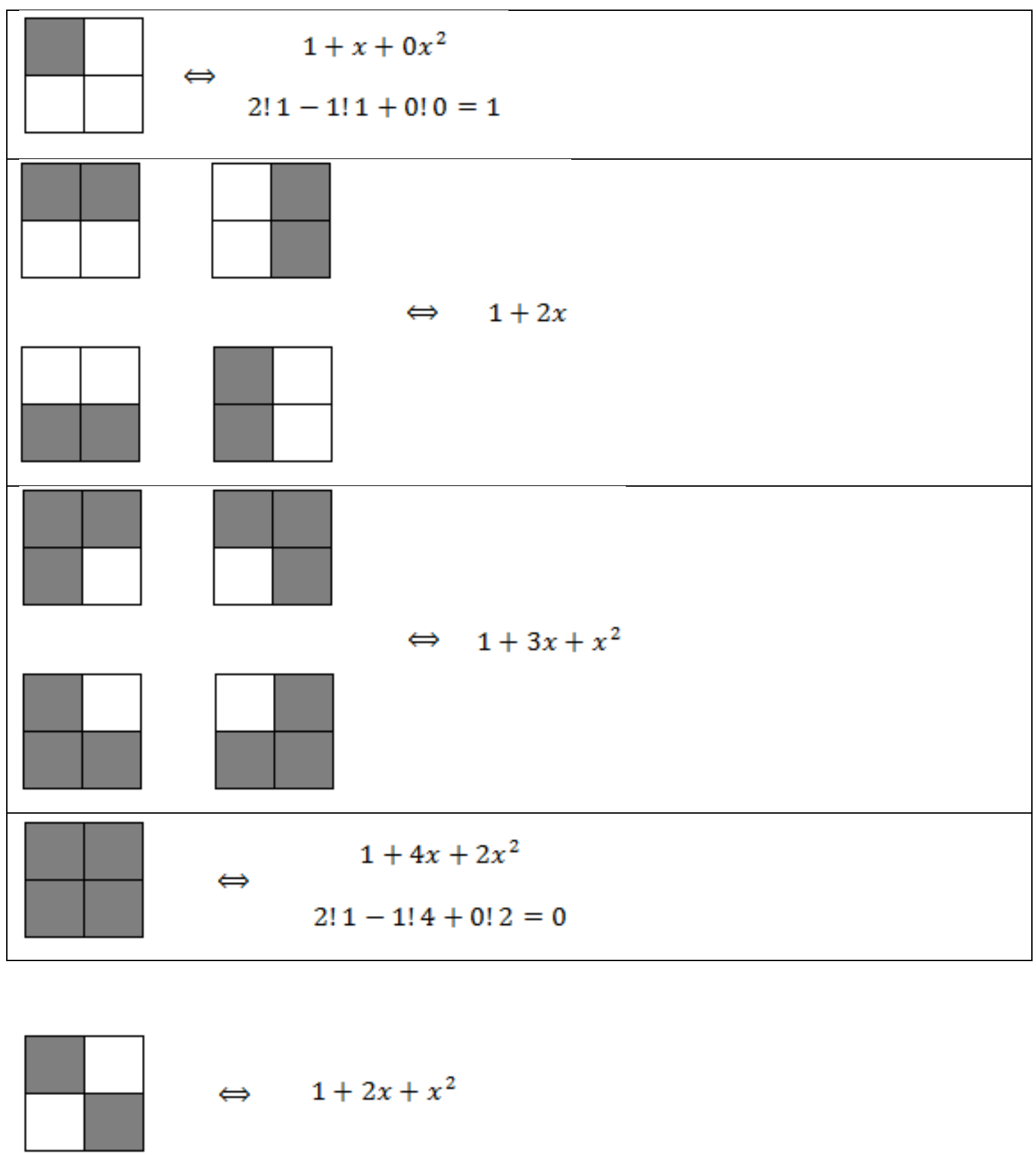

$$
\Leftrightarrow \quad 1+2 x+x^{2}
$$

$\Uparrow$

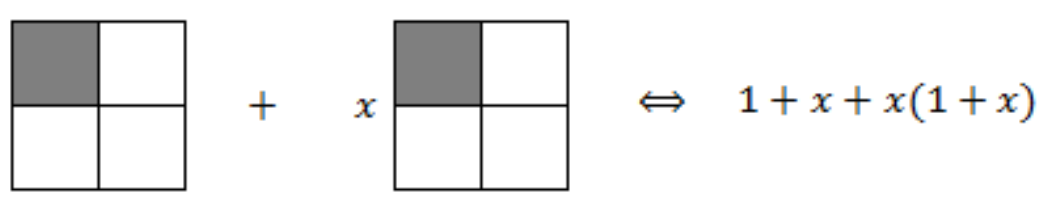


O método fundamental de redução do polinômio torre é justificado pelo fato de que, escolhido um quadrado hachurado $q$ ou o quadrado $q$ ocorre na seleção dos demais $k$ quadrados e, portanto, os $k-1$ quadrados distintos de $q$ não podem ocorrer na mesma linha e coluna determinada por $q$, ou o quadrado $q$ não aparece na seleção dos $k$ quadrados e isto significa que $q$ não necessita ser hachurado.

Isto explica que o polinômio torre, associado a

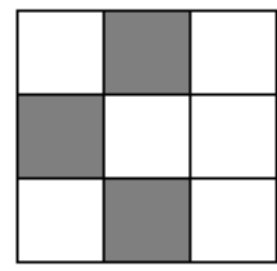

é igual a soma do polinômio torre associado a

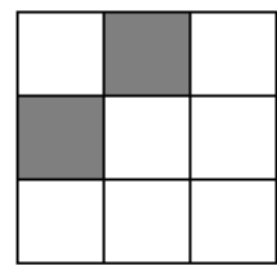

com o polinômio torre associado a

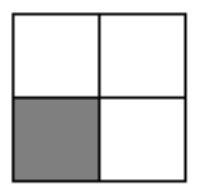

multiplicado por $x$, isto é, é igual a

$$
\left(1+2 x+x^{2}\right)+x(1+x)=1+3 x+2 x^{2}+0 x^{3}
$$

$\mathrm{O}$ valor $(3 ! 1-2 ! 3+1 ! 2-0$ ! 0$)$ calcula o número de permutações do conjunto $\{1,2,3\}$ sujeitas às restrições

$$
f(1) \neq 2 \quad f(2) \neq 1 \quad f(3) \neq 2
$$

e o cálculo é justificado pelo teorema da inclusão e exclusão tendo em vista que pelo teorema, o conjunto $U$ é o conjunto de todas as funções injetivas e sobrejetivas $f \operatorname{com} D(f)=R(f)=$ $\{1,2,3\}$. 
$A \subset U$ é o conjunto das funções $f \operatorname{com} f(1)=2$,

$B \subset U$ é o conjunto das funções $f \operatorname{com} f(2)=1$,

$C \subset U$ é o conjunto das funções $f \operatorname{com} f(3)=2$

e assim

$$
\begin{gathered}
|A|=|B|=|C|=2 ! \\
|A \cap B|=|B \cap C|=1 ! \\
|A \cap C|=0 \\
|A \cap B \cap C|=0
\end{gathered}
$$

o que mostra que a resposta ao problema é dado por

$$
\begin{gathered}
\left|A^{c} \cap B^{c} \cap C^{C}\right|=\left|(A \cup B \cup C)^{C}\right| \\
=|U|-|A \cup B \cup C| \\
=|U|-[|A|+|B|+|C|]+[|A \cap B|+|A \cap C|+|B \cap C|]-|A \cap B \cap C| \\
=3 !-3 \cdot 2 !+2 \cdot 1 !-0 \cdot 0 !
\end{gathered}
$$

que é igual ao resultado com a utilização dos polinômios-torre.

\subsection{NÚMERO DE FUNÇÕES CRESCENTES OU DECRESCENTES}

O número de funções crescentes (decrescentes) $f: D(f)=\{1,2, \ldots, n\} \rightarrow\{0,1,2, \ldots, 9\}$ é igual ao número de sequências crescentes (decrescentes) com comprimento $n$ em que ocorram apenas os dígitos $0,1,2, \ldots, 9$ que é igual ao numero de soluções não negativas de

$$
x_{0}+x_{1}+x_{2}+\cdots+x_{9}=n
$$

em que $x_{0}$ indica o número de algarismos zeros presentes na sequência, $x_{1}$ indica o número de algarismos, uns presentes na sequência e, assim por diante, $x_{9}$ indica o número de algarismos nove presentes na sequência crescente. 
Para calcular o número de soluções inteiras não negativas da equação

$$
x_{0}+x_{1}+x_{2}+\cdots+x_{9}=n
$$

inicialmente calculamos o número de soluções inteiras não negativas de

$$
x+y=3 \quad x, y \in Z \quad x \geq 0 \quad y \geq 0
$$

que é igual ao número de sequências binárias de comprimento 4 (quatro) com três dígitos iguais a um e um digito igual a zero: por exemplo,

\begin{tabular}{|ll}
0111 & indica que $x=0$ e $y=3$ \\
1011 & indica que $x=1$ e $y=2$ \\
1101 & indica que $x=2$ e $y=1$ \\
1110 & indica que $x=3$ e $y=0$
\end{tabular}

que são as $\left(\begin{array}{l}4 \\ 1\end{array}\right)$ soluções inteiras não negativas de $x+y=3$, analogamente, para calcular o número de soluções inteiras não negativas de

$$
x+y+z=7
$$

Basta calcular o número de sequências binárias com comprimento 9 (nove) com sete dígitos iguais a um e dois dígitos iguais a zero: por exemplo,

$$
\begin{aligned}
& 110111101 \quad \text { indica que } x=2, y=4 \text { e } z=1 \\
& 111001111 \quad \text { indica que } x=3, y=0 \text { e } z=4
\end{aligned}
$$

e o número de tais sequências é igual a $\left(\begin{array}{l}9 \\ 2\end{array}\right)=\left(\begin{array}{l}9 \\ 7\end{array}\right)$ tendo em vista que dos nove dígitos há de se fazer a escolha da posição dos dois dígitos iguais a zero. Portanto, o número de soluções inteiras não negativas de

$$
x+y=n \quad x, y \in Z \quad x \geq 0 \quad y \geq 0
$$

é igual a $\left(\begin{array}{c}n+1 \\ 1\end{array}\right)$; o número de soluções inteiras não negativas de 


$$
x+y+z=n \quad x, y, z \in Z \quad x \geq 0 \quad y \geq 0 \quad z \geq 0
$$

é igual a $\left(\begin{array}{c}n+2 \\ 2\end{array}\right)$; o número de soluções inteiras não negativas de

$$
w+x+y+z=n \quad w, x, y, z \in Z \quad w \geq 0 \quad x \geq 0 \quad y \geq 0 \quad z \geq 0
$$

é igual a $\left(\begin{array}{c}n+3 \\ 3\end{array}\right)$. Lembrando que o número de soluções inteiras não negativas $x=n$ é igual a $\left(\begin{array}{c}n+0 \\ 0\end{array}\right)=1$ e que a série geométrica

$$
\frac{1}{1-t}=\sum_{n=0}^{\infty} t^{n}=1+t+t^{2}+\cdots t^{n}+\cdots
$$

indica que o coeficiente um de $t^{n}$ é o número de soluções inteiras não negativas de $x=n$ então

$$
\frac{1}{(1-t)^{2}}=\sum_{0}^{\infty}\left(\begin{array}{c}
n+1 \\
1
\end{array}\right) t^{n}
$$

e $\left(\begin{array}{c}n+1 \\ 1\end{array}\right)$, coeficiente de $t^{n}$, é o número de soluções inteiras não negativas de

$$
x+y=n
$$

e então como

$$
\frac{1}{(1-t)^{3}}=\sum_{0}^{\infty}\left(\begin{array}{c}
n+2 \\
2
\end{array}\right) t^{n}
$$

$\left(\begin{array}{c}n+2 \\ 2\end{array}\right)$, coeficiente de $t^{n}$, é o número de soluções inteiras não negativas de $x+y+z=n$

Em vista do exposto, $\left(\begin{array}{c}n+9 \\ 9\end{array}\right)$ coeficiente de $t^{n}$ em

$$
\frac{1}{(1-t)^{10}}=\sum_{0}^{\infty}\left(\begin{array}{c}
n+9 \\
9
\end{array}\right) t^{n}
$$


é o número de soluções inteiras não negativas de $x_{0}+x_{1}+x_{2}+\cdots+x_{9}=n$ que é igual ao número de funções crescentes (decrescentes) $f: D(f)=\{1,2, \ldots, n\} \rightarrow\{0,1,2, \ldots, 9\}$ e que é igual ao número de sequências crescentes (decrescentes) com comprimento $n$ em que ocorrem os algarismos decimais $0,1, \ldots, 9$.

\section{Exemplo 8.}

O número de senhas bancárias, crescentes ou decrescentes, com 7 (sete) dígitos em que ocorrem $n$ algarismos decimais $0,1, \ldots, 9$ é igual ao número de soluções inteiras não negativas de

$$
x_{0}+x_{1}+x_{2}+\cdots+x_{9}=7
$$

que é igual a $\left(\begin{array}{c}7+9 \\ 9\end{array}\right)$

\section{Exemplo 9.}

O número de cartões nacionais de saúde crescentes ou decrescentes com 15 dígitos em que ocorrem os algarismos decimais $0,1, \ldots, 9$ é igual ao número de soluções inteiras não negativas de

$$
x_{0}+x_{1}+x_{2}+\cdots+x_{9}=15
$$

que é igual a $\left(\begin{array}{c}15+9 \\ 9\end{array}\right)$

Existe uma correspondência biunívoca canônica entre o conjunto das soluções inteiras não negativas de

$$
x+y+z=10 \quad x \geq 2 \quad y \geq 3 \quad z \geq 1 \quad x, y, z \in Z
$$

e o conjunto de soluções inteiras não negativas de

$$
x+y+z=10-2-3-1=4 \quad x \geq 0 \quad y \geq 0 \quad z \geq 0 \quad x, y, z \in Z
$$

Portanto a cardinalidade dos dois conjuntos é igual ao número binomial

$$
\left(\begin{array}{l}
6 \\
2
\end{array}\right)=\left(\begin{array}{l}
6 \\
4
\end{array}\right)=15
$$


A generalização deste fato é a existência de uma correspondência biunívoca entre o conjunto das soluções inteiras não negativas de

$$
\begin{gathered}
x_{1}+x_{2}+\cdots+x_{r}=n \\
x_{1} \geq n_{1} x_{2} \geq n_{2} \ldots \geq n_{r} x_{1}, x_{2}, \ldots, x_{r} \in Z
\end{gathered}
$$

$\mathrm{e}$

$$
x_{1}+x_{2}+\cdots+x_{r}=n-n_{1}-n_{2} \cdots-n_{r}
$$

Logo, a cardinalidade dos dois conjuntos é igual ao número binomial

$$
\left(\begin{array}{c}
n-n_{1}-n_{2} \cdots-n_{r}+r-1 \\
r-1
\end{array}\right)
$$

\subsection{PERMUTAÇÕES CAÓTICAS}

Considere a situação:

\section{Exemplo 10.}

Um concurso de dança composto por seis casais tem o improviso como um dos critérios a serem avaliados. Para evitar ensaios, ficou estabelecido que não será permitida a formação de duplas de dançarinos que sejam marido e esposa. De quantas maneiras pode-se organizar esse concurso?

O problema pode ser solucionado mediante a contagem das Permutações Caóticas.

Definição: Uma permutação de $a_{1}, a_{2}, \ldots, a_{n}$ é chamada de caótica ou de desarranjo, quando nenhum dos $a_{i}$ se encontra na posição original, isto é, na $i$-ésima posição.

Considere $D_{n}$ o número permutações caóticas das $n$ letras $a, b, c, d, \ldots$

Se $n=1$, temos que $D_{1}=0$, pois $a$ não pode ocupar sua posição.

Se $n=2$, temos que $D_{2}=1$, pois há uma única forma de desarranjo: $b a$.

Para $n=3$, temos $D_{3}=2: c a b ; b c a$.

Continuando com os casos particulares encontramos $D_{4}=9$ e $D_{5}=44$, mas é evidente a necessidade de dedução matemática para uma lei de formação de $D_{n}$. 
Seja $a, b, c, d, \ldots$ a formação inicial das $n$ letras. Vamos organizá-las de modo que nenhuma ocupe sua posição original, dessa forma para a primeira posição há $n-1$ maneiras de ocupá-la, pois a letra $a$ não figura. Suponhamos que $b$ ocupou a primeira posição, dessa forma $D_{n}$ é o produto do número de variações das demais letras por $n-1$. Se $b$ é a primeira letra, então podemos dividir em dois casos:

- $1^{\circ}$ caso: A segunda letra é o $a$. Então temos que organizar as $n-2$ letras que restam de modo que nenhuma ocupe sua posição original, portanto há $D_{n-2}$ modos de organizá-las.

- $\quad 2^{\circ}$ caso: A segunda letra não é o $a$. Então temos que organizar $n-1$ letras à direita de $b$ e isso pode ser feito em $D_{n-1}$ modos.

Como os casos são disjuntos, temos que, com $b$ na primeira posição, podemos permutar as demais letras de $D_{n-1}+D_{n-2}$ modos, mas como há $n-1$ formas de ocupar a primeira posição, temos que:

$$
D_{n}=(n-1)\left(D_{n-1}+D_{n-2}\right)
$$

Fazendo $n=3$, temos:

$$
D_{3}=2\left(D_{2}+D_{1}\right) \Rightarrow D_{3}=2 D_{2}+2 D_{1}
$$

Reescrevendo a expressão, obtemos:

$$
D_{3}=\left(-D_{2}+3 D_{2}\right)+2 D_{1} \Rightarrow D_{3}-3 D_{2}=-\left(D_{2}-2 D_{1}\right)
$$

De forma análoga para $n=4$ e $n=5$ temos, respectivamente:

$$
\begin{aligned}
& D_{4}-4 D_{3}=-\left(D_{3}-3 D_{2}\right) \\
& D_{5}-5 D_{4}=-\left(D_{4}-4 D_{3}\right)
\end{aligned}
$$

Logo para $n \geq 3$ têm-se:

$$
\begin{aligned}
& D_{4}-4 D_{3}=-\left(D_{3}-3 D_{2}\right) \\
& D_{5}-5 D_{4}=-\left(D_{4}-4 D_{3}\right)
\end{aligned}
$$




$$
D_{n}-n D_{n-1}=-\left(D_{n-1}-(n-1) D_{n-2}\right)
$$

Se multiplicarmos todas as $n-2$ igualdades, teremos:

$$
\begin{gathered}
\left(D_{3}-3 D_{2}\right)\left(D_{4}-4 D_{3}\right) \ldots\left(D_{n}-n D_{n-1}\right) \\
=(-1)^{n-2}\left(D_{2}-2 D_{1}\right)\left(D_{3}-3 D_{2}\right) \ldots\left(D_{n-1}-(n-1) D_{n-2}\right) \\
\quad \Rightarrow D_{n}-n D_{n-1}=(-1)^{n-2}\left(D_{2}-2 D_{1}\right)
\end{gathered}
$$

Como $(-1)^{n-2}=(-1)^{n}$, para qualquer $n$ inteiro e $D_{2}-2 D_{1}=1$ então,

$$
D_{n}-n D_{n-1}=(-1)^{n} \Rightarrow D_{n}=n D_{n-1}+(-1)^{n}, \quad \forall n \geq 3
$$

Decorre da igualdade que:

$$
\begin{gathered}
D_{3}=3 D_{2}-1 \\
D_{4}=4 D_{3}+1=4\left(3 D_{2}-1\right)+1=4.3 D_{2}-4+1=4.3-4+1 \\
D_{5}=5 D_{4}-1=5(4.3-4+1)-1=5.4 .3-5.4+5-1
\end{gathered}
$$

Observe que:

$$
5.4 .3-5.4+5-1=\frac{5 !}{2 !}-\frac{5 !}{3 !}+\frac{5 !}{4 !}-\frac{5 !}{5 !}=5 !\left(\frac{1}{2 !}-\frac{1}{3 !}+\frac{1}{4 !}-\frac{1}{5 !}\right)
$$

ou seja, $D_{5}=5 !\left(\frac{1}{2 !}-\frac{1}{3 !}+\frac{1}{4 !}-\frac{1}{5 !}\right)$

Provemos por indução que

$$
D_{n}=n !\left(\frac{1}{2 !}-\frac{1}{3 !}+\frac{1}{4 !}-\frac{1}{5 !}+\cdots+(-1)^{n} \frac{1}{n !}\right), \quad \forall n \geq 2
$$

De fato, para $n=2$, tem-se:

$$
D_{2}=2 !\left(\frac{1}{2 !}\right)=1
$$

Suponhamos, por hipótese de indução, que seja válido para $n-1$, isto é: 


$$
D_{n-1}=(n-1) !\left(\frac{1}{2 !}-\frac{1}{3 !}+\frac{1}{4 !}-\frac{1}{5 !}+\cdots+(-1)^{n-1} \frac{1}{(n-1) !}\right)
$$

Multiplicando ambos os membros da igualdade por $n$, fica:

$$
n D_{n-1}=n(n-1) !\left(\frac{1}{2 !}-\frac{1}{3 !}+\frac{1}{4 !}-\frac{1}{5 !}+\cdots+(-1)^{n-1} \frac{1}{(n-1) !}\right)
$$

Como

$$
D_{n}=n D_{n-1}+(-1)^{n} \Rightarrow n D_{n-1}=D_{n}-(-1)^{n}
$$

$\log 0$

$$
D_{n}-(-1)^{n}=n(n-1) !\left(\frac{1}{2 !}-\frac{1}{3 !}+\frac{1}{4 !}-\frac{1}{5 !}+\cdots+(-1)^{n-1} \frac{1}{(n-1) !}\right)
$$

isto é,

$$
\begin{gathered}
D_{n}=n(n-1) !\left(\frac{1}{2 !}-\frac{1}{3 !}+\frac{1}{4 !}-\frac{1}{5 !}+\cdots+(-1)^{n-1} \frac{1}{(n-1) !}\right)+(-1)^{n} \\
D_{n}=n !\left(\frac{1}{2 !}-\frac{1}{3 !}+\frac{1}{4 !}-\frac{1}{5 !}+\cdots+(-1)^{n} \frac{1}{n !}\right)
\end{gathered}
$$

Como $D_{1}=0$ podemos ajustar escrevendo:

$$
D_{n}=n !\left(\frac{1}{0 !}-\frac{1}{1 !}+\frac{1}{2 !}-\frac{1}{3 !}+\frac{1}{4 !}-\frac{1}{5 !}+\cdots+(-1)^{n} \frac{1}{n !}\right) \quad \forall n \geq 1
$$

Voltemos ao exemplo

Se considerarmos os homens como sendo as posições $(1,2, \ldots n)$ e as mulheres as letras $(a, b, c, \ldots)$, então a formação

$$
\left(\begin{array}{l}
123456 \\
\text { acbedf }
\end{array}\right)
$$

não é permitida, pois a mulher (a) está com seu marido (1) e o homem (6) está com sua esposa $(f)$. Dessa forma, a solução para o problema é:

$$
D_{6}=6 !\left(\frac{1}{0 !}-\frac{1}{1 !}+\frac{1}{2 !}-\frac{1}{3 !}+\frac{1}{4 !}-\frac{1}{5 !}+\frac{1}{6 !}\right)=265 \text { maneiras }
$$




\subsection{NÚMEROS DE FUNÇÕES COM RESTRIÇÕES}

O cálculo do número de funções $f: D(f)=\{1,2,3,4\} \rightarrow\{1,2,3,4,5,6,7,8,9\}$ com $R(f) \subset\{1,2,3,4,5,6,7,8,9\}$ sujeitas às restrições

$$
f(1) \neq f(2) \quad f(1) \neq f(4) \quad f(2) \neq f(3) \quad f(3) \neq f(4)
$$

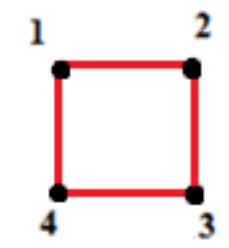

é a soma do número de funções $f$ sujeitas às condições acima em que $f(2)=f(4)$, que é igual a

$$
9 \cdot 8 \cdot 8
$$

com o número de funções $f$ sujeitas às condições acima em que $f(2) \neq f(4)$ que é igual a

$$
9 \cdot 8 \cdot 7 \cdot 7
$$

No caso de $R(f)$ estar contido em um conjunto com $n$ elementos, o número de funções $f$ sujeitas às restrições dadas é igual a $n(n-1)^{2}+n(n-1)(n-2)^{2}$ que é denominado polinômio cromático para o problema em questão.

Um raciocínio análogo permite justificar o método fundamental de redução do polinômio cromático associado a problemas de contagem de funções $f$ sujeitas a restrições

$$
f(1) \neq f(2) \quad f(1) \neq f(4) \quad f(2) \neq f(3) \quad f(3) \neq f(4)
$$

em que $f: D(f)=\{1,2,3,4\} \rightarrow\{1,2, \ldots, n\}$, isto é, $R(f) \subset\{1,2, \ldots, n\}$.

O número de funções $f$ tais que $D(f)=\{1,2,3,4\}$ e $R(f) \subset\{1,2, \ldots, n\}$ sujeitas as condições

$$
f(1) \neq f(4) \quad f(1) \neq f(2) \quad f(3) \neq f(4)
$$




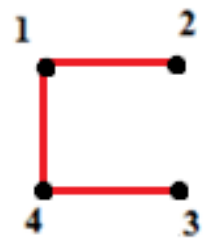

é a soma das possíveis funções $f$ em que

$$
f(1) \neq f(4) \quad f(1) \neq f(2) \quad f(3) \neq f(4) \quad f(2)=f(3)
$$

com o número das funções $f$ em que

$$
f(1) \neq f(4) \quad f(1) \neq f(2) \quad f(3) \neq f(4) \quad f(2) \neq f(3)
$$

o que justifica que o polinômio cromático do grafo

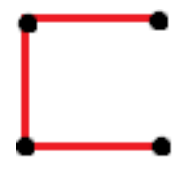

é a soma do polinômio cromático ao grafo

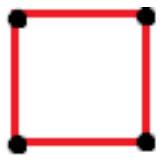

com o polinômio cromático associado ao grafo

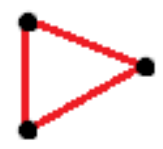

isto é,

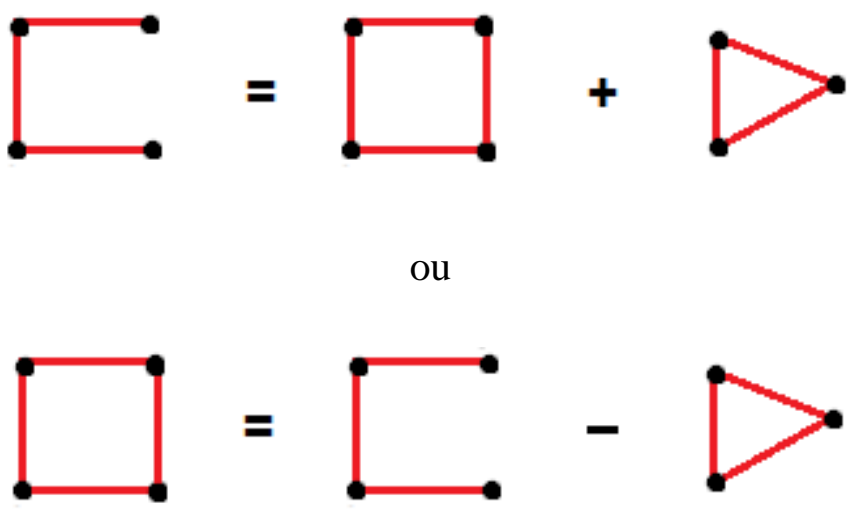




\section{Exemplo 11.}

(OBMEP-2013) Paulo tem tintas de quatro cores diferentes. De quantas maneiras ele pode pintar as regiões da bandeira da figura, cada uma com uma única cor, de modo que cada cor apareça pelo menos uma vez e que regiões adjacentes sejam pintadas com cores diferentes?
A) 336
B) 420
C) 576
D) 864
E) 972

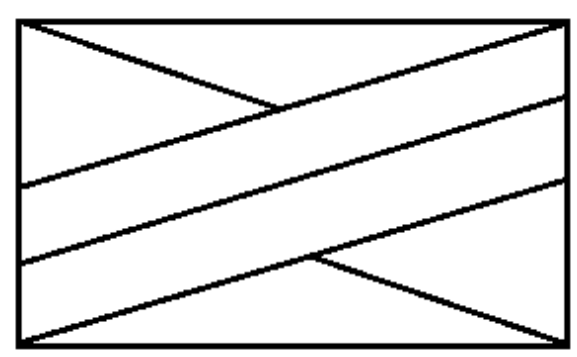

Solução:

Um grafo correspondente à formação da bandeira pode ser representado a seguir. Os vértices, numerados de 1 a 6 , representam as regiões da bandeira e as arestas ligam as regiões que não podem possuir mesma cor.
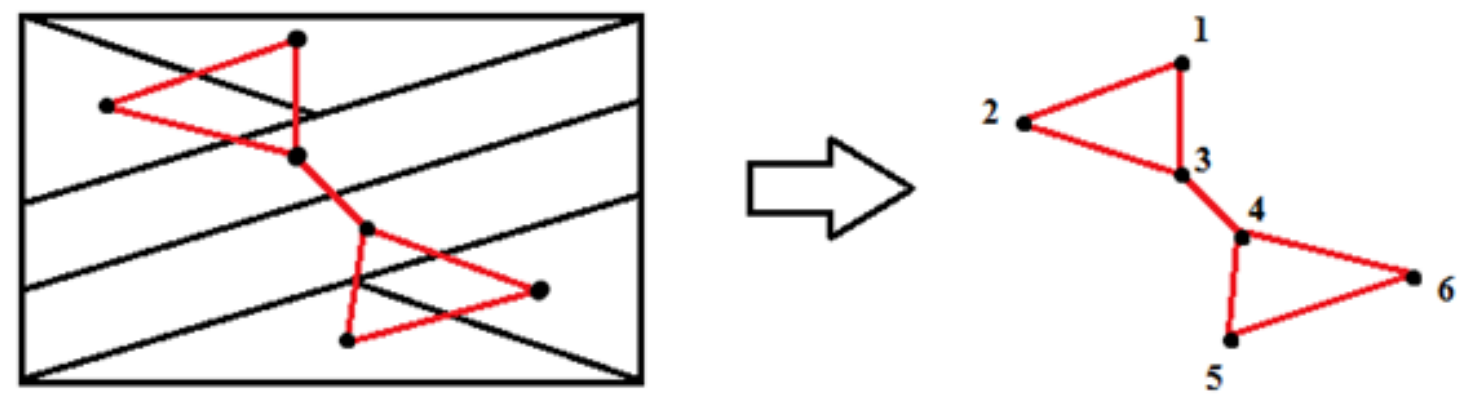

O objetivo é obter uma formação mais simples, ou seja, a decomposição do grafo original.

Se removermos a aresta (13), temos a seguinte decomposição:
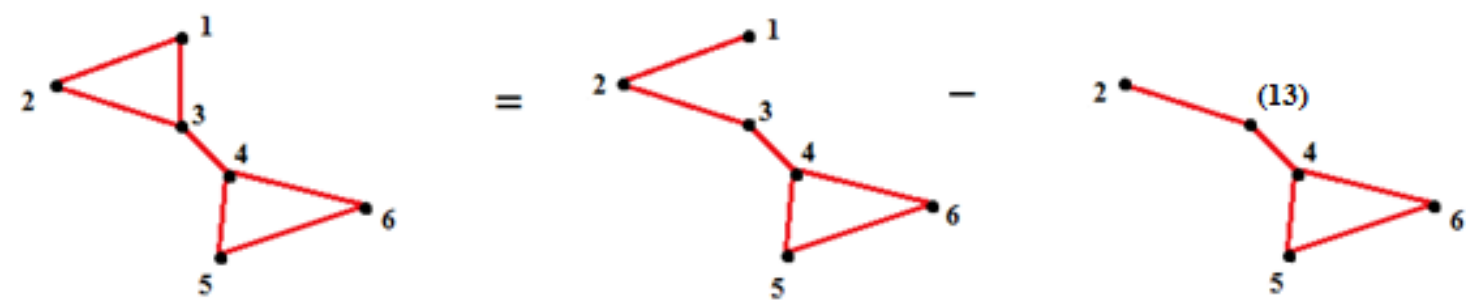

removendo agora a aresta (46), obtemos uma decomposição completa do grafo. 

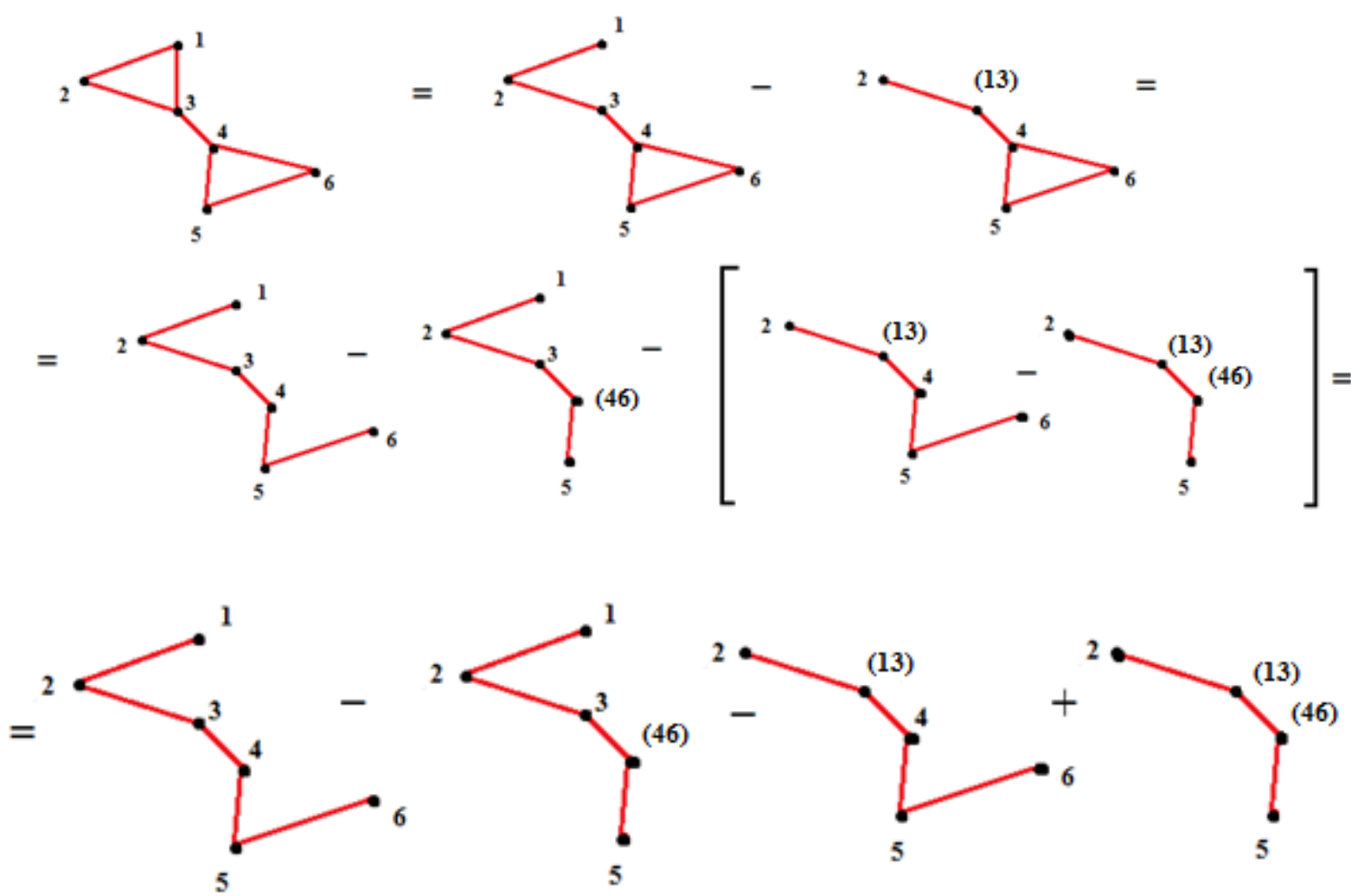

O polinômio que fornece o número de maneiras de colorir a bandeira com $n$ cores pode ser obtido por:
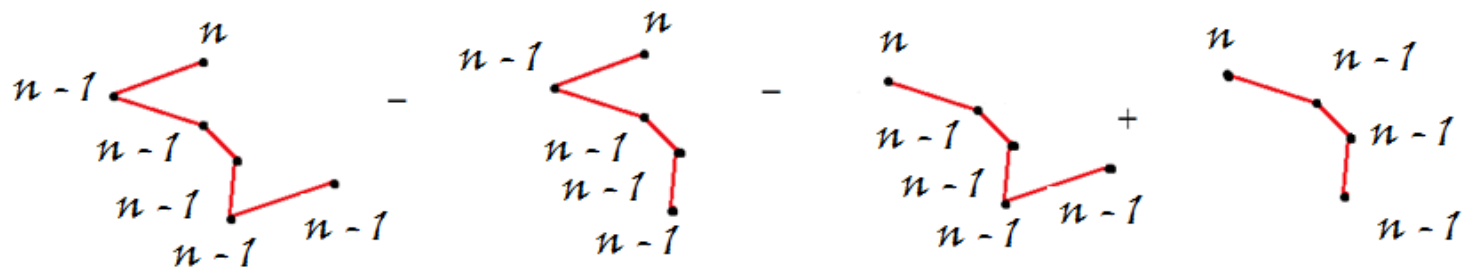

ou seja, $p(n)=n(n-1)^{5}-2 n(n-1)^{4}+n(n-1)^{3}$.

Com $n=4$, temos $p(4)=432$, contudo devemos descartar as formas de pintar a bandeira com três cores, já que uma das condições iniciais é que devem ser utilizadas todas as cores. Assim, se considerarmos as cores (ABCD) podem ocorrer os casos em que não são utilizadas as cores A, B, C, ou D. Dessa forma, a solução para o problema será:

$$
p(4)-4 \cdot p(3)=432-4 \cdot 24=336
$$

Alternativa A. 
Exemplo 12.

$$
f: D(f)=\{1,2, \ldots, 9\} \rightarrow R(f)=\{a, b, c, d, e\}
$$

Supondo que

$$
\begin{aligned}
& f(1) \neq f(5) \\
& f(1) \neq f(7) \\
& f(5) \neq f(7) \\
& f(7) \neq f(9)
\end{aligned}
$$

Um grafo representante para essas condições pode ser:
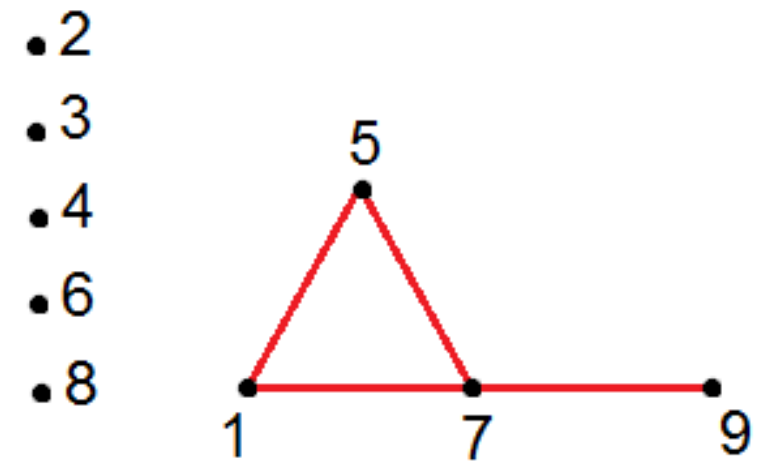

removendo a aresta (57), obtemos a decomposição
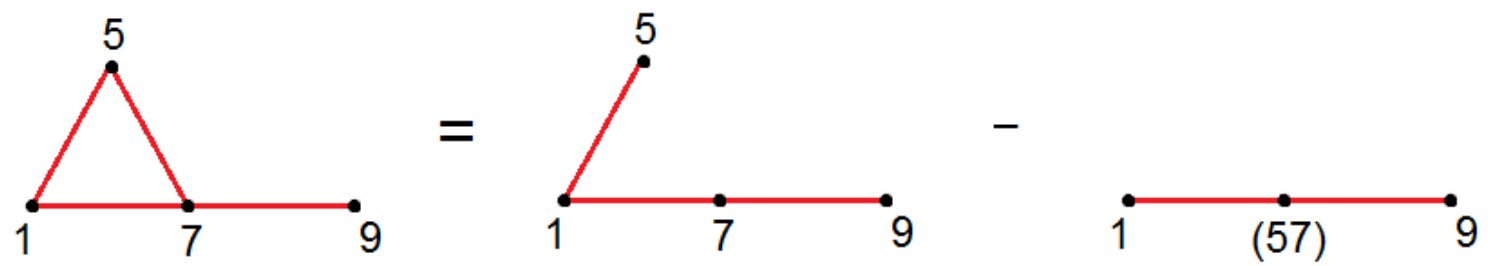

Dessa forma, o polinômio cromático, pode ser obtido por: 

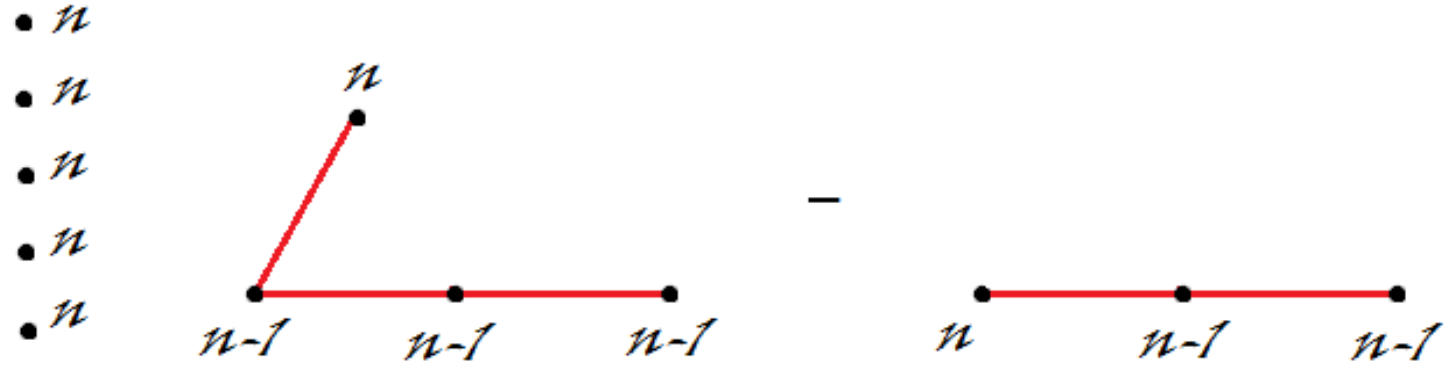

$$
p(n)=n^{5} \cdot n \cdot(n-1)^{3}-n \cdot(n-1)^{2}=n^{6} \cdot(n-1)^{2} \cdot(n-2)
$$

Quando $n=5, p(5)=5^{6} \cdot 4^{2} \cdot 3=750000$.

A sequência representada pelo diagrama,

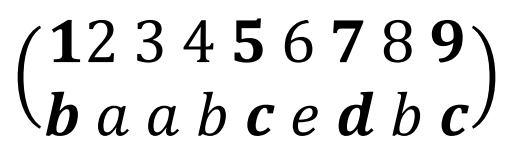

é uma das 750000 sequências que satisfazem as condições.

\subsection{NÚMERO MULTINOMIAL}

O número de partições do conjunto $X=\{1,2,3, \ldots, n\}$ da forma $\left(X_{1}, X_{2}, \ldots, X_{r}\right)$ em que

$$
\left|X_{1}\right|=n_{1},\left|X_{2}\right|=n_{2}, \ldots,\left|X_{r}\right|=n_{r} ; n=n_{1}+n_{2}+\cdots+n_{r}
$$

é dado por

$$
\left(\begin{array}{c}
n \\
n_{1} n_{2} \ldots n_{r}
\end{array}\right)=\frac{n !}{n_{1} ! n_{2} ! \ldots n_{r} !}
$$

A expansão multinominal de

$$
(x+y+z)^{n}
$$

é dada por

$$
(x+y+z)^{n}=\sum_{n=n_{1}+n_{2}+n_{3}}\left(\begin{array}{c}
n \\
n_{1} n_{2} n_{3}
\end{array}\right) x^{n_{1}} y^{n_{2}} z^{n_{3}}
$$

Para compreender, retornaremos ao binômio do exemplo 4 no capítulo 3. 


$$
(a+c)^{4}=1 a^{4}+4 a^{3} c+6 a^{2} c^{2}+4 a c^{3}+1 c^{4}
$$

\begin{tabular}{|c|c|c|}
\hline Termo & $\begin{array}{c}\text { Coeficiente igual ao número } \\
\text { de anagramas da parte } \\
\text { literal }\end{array}$ & Número multinominal \\
\hline $1 a^{4}=1$ aaaa & 1 & $\left(\begin{array}{l}4 \\
4\end{array}\right)=1$ \\
\hline $4 a^{3} c=4 a a a c$ & 4 & $\left(\begin{array}{c}4 \\
3\end{array}\right)=\frac{4 !}{3 ! 1 !}=4$ \\
\hline $6 a^{2} c^{2}=6 a a c c$ & 6 & $\left(\begin{array}{c}4 \\
2\end{array}\right)=\frac{4 !}{2 ! 2 !}=6$ \\
\hline $4 a c^{3}=4 a c c c$ & 4 & $\left(\begin{array}{c}4 \\
13\end{array}\right)=\frac{4 !}{1 ! 3 !}=4$ \\
\hline $1 c^{4}=1 c c c c$ & 1 & $\left(\begin{array}{l}4 \\
4\end{array}\right)=1$ \\
\hline
\end{tabular}

Dessa forma, podemos expandir

$$
(x+y+z)^{3}
$$

em

\begin{tabular}{|c|c|c|}
\hline Termo & $\begin{array}{c}\text { Coeficiente igual ao número } \\
\text { de anagramas da parte } \\
\text { literal }\end{array}$ & Número multinominal \\
\hline $1 x^{3}=1 x x x$ & 1 & $\left(\begin{array}{l}3 \\
3\end{array}\right)=1$ \\
\hline $1 y^{3}=1 y y y$ & 1 & $\left(\begin{array}{l}3 \\
3\end{array}\right)=1$ \\
\hline $1 z^{3}=1 z z z$ & 1 & $\left(\begin{array}{l}3 \\
3\end{array}\right)=1$ \\
\hline $3 x^{2} y=3 x x y$ & 3 & $\left(\begin{array}{c}3 \\
2\end{array}\right)=\frac{3 !}{2 ! 1 !}=3$ \\
\hline
\end{tabular}




\begin{tabular}{|c|c|c|}
\hline $3 x y^{2}=3 x y y$ & 3 & $\left(\begin{array}{c}3 \\
12\end{array}\right)=\frac{3 !}{1 ! 2 !}=3$ \\
\hline $3 x^{2} z=3 x x z$ & 3 & $\left(\begin{array}{c}3 \\
21\end{array}\right)=\frac{3 !}{2 ! 1 !}=3$ \\
\hline $3 x z^{2}=3 x z z$ & 3 & $\left(\begin{array}{c}3 \\
12\end{array}\right)=\frac{3 !}{1 ! 2 !}=3$ \\
\hline $3 y^{2} z=3 y y z$ & 3 & $\left(\begin{array}{c}3 \\
2 \\
2\end{array}\right)=\frac{3 !}{2 ! 1 !}=3$ \\
\hline $3 y z^{2}=3 y z z$ & 3 & $\left(\begin{array}{c}3 \\
12\end{array}\right)=\frac{3 !}{1 ! 2 !}=3$ \\
\hline $6 x y z$ & 6 & 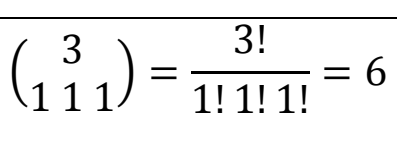 \\
\hline
\end{tabular}

$(x+y+z)^{3}=x^{3}+3 x y^{2}+6 x y z+3 x z^{2}+y^{3}+3 y x^{2}+3 y z^{2}+z^{3}+3 z x^{2}+3 z y^{2}$

\subsection{NÚMEROS DE STIRLING DE PRIMEIRA ESPÉCIE}

Antes de iniciarmos com os números de Stirling de primeira espécie, faz-se necessária uma breve apresentação do conceito sobre ciclos em uma permutação.

\section{Exemplo 13.}

Considere o conjunto $X=\{1,2,3,4\}$, temos que $\left|S_{4}\right|=4 !=24$, das quais a formação 1243 trata-se de uma das permutações, em que o algarismo 1 está no lugar do próprio 1; o 2 está no lugar do próprio 2; o 4 está no lugar do 3; e o 3 está no lugar do 4. Esse exemplo também pode ser representado pelo diagrama

$$
\sigma=\left(\begin{array}{llll}
1 & 2 & 3 & 4 \\
1 & 2 & 4 & 3
\end{array}\right)
$$

ou ainda, por 


$$
1 \rightarrow 1 \quad 1 \rightarrow 1
$$

$2 \rightarrow 2$ equivalente a $2 \rightarrow 2$

$$
3 \rightarrow 4 \quad 3 \rightarrow 4 \rightarrow 3
$$

$4 \rightarrow 3$

que em notação de ciclos representamos por

$$
(1)(2)(34)
$$

Nesse exemplo, temos 3 ciclos.

Considere o seguinte problema:

De quantas maneiras quatro pessoas podem ocupar duas mesas sem que nenhuma fique vazia? E se forem três mesas?

Esse problema pode ser resolvido contando o número de 2 -ciclos e 3 -ciclos presentes no conjunto $X=\{a, b, c, d\}$, em que os elementos do conjunto representam as pessoas da situação. A distribuição pode ser organizada da seguinte maneira:

- Para duas mesas, ou seja, 2 - ciclos

\begin{tabular}{|l|l|}
\hline$(a)(b c d)$ & Pessoa $a$ senta-se sozinha. Pessoas $b, c$ e $d$ sentam-se juntas. \\
\hline$(a)(b d c)$ & Pessoa $a$ senta-se sozinha. Pessoas $b, d$ e $c$ sentam-se juntas. \\
\hline$(b)(a c d)$ & Pessoa $b$ senta-se sozinha. Pessoas $a, c$ e $d$ sentam-se juntas. \\
\hline$(b)(a d c)$ & Pessoa $b$ senta-se sozinha. Pessoas $a, d$ e $c$ sentam-se juntas. \\
\hline$(c)(b a d)$ & Pessoa $c$ senta-se sozinha. Pessoas $b, a$ e $d$ sentam-se juntas. \\
\hline$(c)(b d a)$ & Pessoa $c$ senta-se sozinha. Pessoas $b, d$ e $a$ sentam-se juntas. \\
\hline$(d)(a b c)$ & Pessoa $d$ senta-se sozinha. Pessoas $a, b$ e $c$ sentam-se juntas. \\
\hline$(d)(a c b)$ & Pessoa $d$ senta-se sozinha. Pessoas $a, c$ e $b$ sentam-se juntas. \\
\hline$(a b)(c d)$ & Pessoas $a$ e $b$ sentam-se numa mesa, $c$ e $d$ em outra. \\
\hline$(a c)(b d)$ & Pessoas $a$ e $c$ sentam-se numa mesa, $b$ e $d$ em outra. \\
\hline$(a d)(b c)$ & Pessoas $a$ e $d$ sentam-se numa mesa, $b$ e $c$ em outra. \\
\hline
\end{tabular}


Nesse caso, para $|X|=4$, há 11 maneiras de ocupar as duas mesas.

- Para três mesas, ou seja, 3 - ciclos

\begin{tabular}{|l|l|}
\hline$(a)(b)(c d)$ & Pessoas $a$ e $b$ sentam-se sozinhas, pessoas $c$ e $d$ sentam-se juntas. \\
\hline$(a)(c)(b d)$ & Pessoas $a$ e $c$ sentam-se sozinhas, pessoas $b$ e $d$ sentam-se juntas. \\
\hline$(a)(d)(b c)$ & Pessoas $a$ e $d$ sentam-se sozinhas, pessoas $b$ e $c$ sentam-se juntas. \\
\hline$(b)(c)(a d)$ & Pessoas $b$ e $c$ sentam-se sozinhas, pessoas $a$ e $d$ sentam-se juntas. \\
\hline$(b)(d)(a c)$ & Pessoas $b$ e $d$ sentam-se sozinhas, pessoas $a$ e $c$ sentam-se juntas. \\
\hline$(c)(d)(a b)$ & Pessoas $c$ e $d$ sentam-se sozinhas, pessoas $a$ e $b$ sentam-se juntas. \\
\hline
\end{tabular}

Desse modo, para $|X|=4$, há 6 maneiras de ocupar as três mesas.

Agora podemos definir os números de Stirling de primeira espécie.

Sejam $n, k \in N$, com $n \geq k \geq 1$. A notação $c(n, k)$ indica o número de maneiras de se arranjar $n$ elementos em $k$-ciclos disjuntos ou, como no exemplo acima, o número de maneira de organizar $n$ pessoas em $k$ mesas idênticas com pelo menos uma pessoa por mesa.

Os números de Stirling de primeira espécie respeitam a relação de recorrência

$$
c(n, k)=\left[\begin{array}{l}
n \\
k
\end{array}\right]=c(n-1, k-1)+(n-1) c(n-1, k)
$$

visto que ou o elemento $n$ forma um ciclo e os demais $1,2, \ldots, n-1$ formam $k-1$ ciclos o que resulta em $c(n-1, k-1)$ permutações de $1,2, \ldots, n$ em $k$ ciclos ou o elemento $n$ não forma um ciclo e os demais $1,2, \ldots, n-1$ formam $k$ ciclos que resulta em $c(n-1, k)$ ciclos e em cada um destes $c(n-1, k)$ ciclos o elemento $n$ é adicionada em seguida a cada um, o que pode ser feito em $n-1$ modos.

Com a relação de recorrência é possível construir a tabela com os números de Stirling de primeira espécie. 


\begin{tabular}{|c|c|c|c|c|c|c|c|c|c|}
\hline \multicolumn{1}{|c|}{$c(n, 0)$} & $c(n, 1)$ & $c(n, 2)$ & $c(n, 3)$ & $c(n, 4)$ & $c(n, 5)$ & $c(n, 6)$ & $c(n, 7)$ & $c(n, 8)$ \\
\hline 0 & 1 & & & & & & & & \\
\hline 1 & 0 & 1 & & & & & & & \\
\hline 2 & 0 & 1 & 1 & & & & & & \\
\hline 3 & 0 & 2 & 3 & 1 & & & & & \\
\hline 4 & 0 & 6 & 11 & 6 & 1 & & & & \\
\hline 5 & 0 & 24 & 50 & 35 & 10 & 1 & & & \\
\hline 6 & 0 & 120 & 274 & 225 & 85 & 15 & 1 & & \\
\hline 7 & 0 & 720 & 1764 & 1624 & 735 & 175 & 21 & 1 & \\
\hline 8 & 0 & 5040 & 13068 & 13132 & 6769 & 1960 & 322 & 28 & 1 \\
\hline
\end{tabular}

Note que é possível completar a linha $n=9$ utilizando-se dos valores presentes na linha $n=8$.

Pelo esquema a seguir, podemos compreender como isso se torna possível.

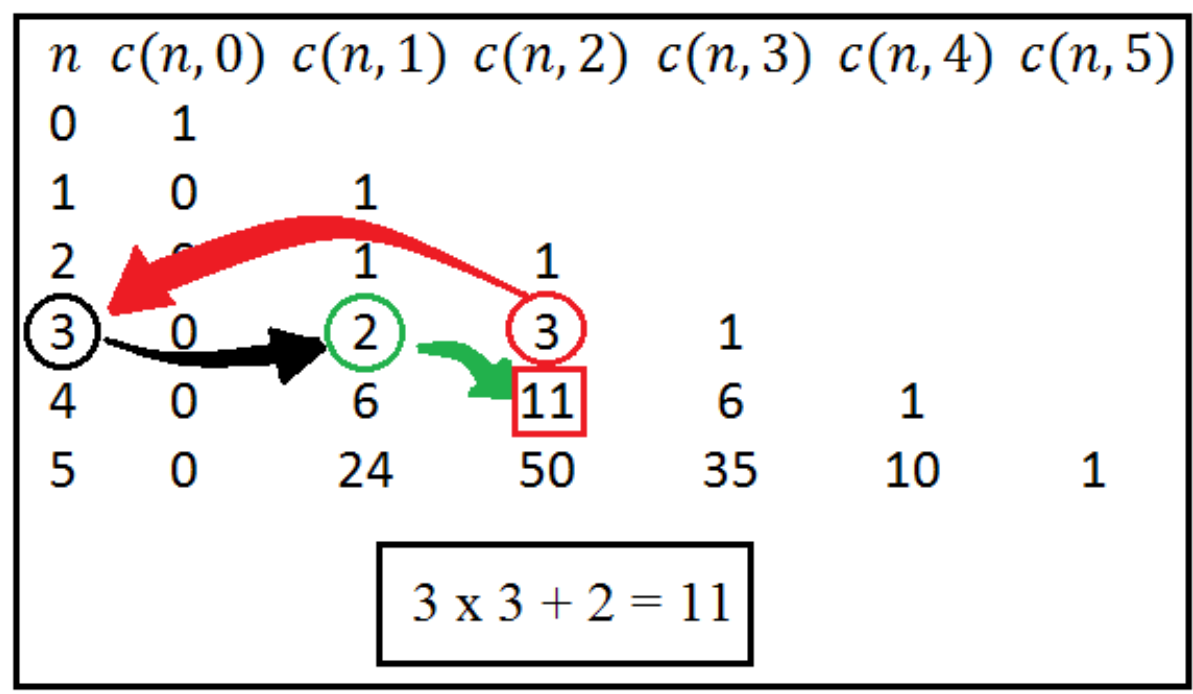

\subsection{NÚMEROS DE STIRLING DE SEGUNDA ESPÉCIE}

O número de partições disjuntas e não vazias de $\{1,2, \ldots, n\}$ é dado pelo número de Stirling de segunda espécie $S(n, k)$. Esses números satisfazem à fórmula de recorrência:

$$
S(n, k)=S(n-1, k-1)+k S(n-1, k), \forall n>0, \forall k \leq n
$$


Considere um $n$-conjunto $X$, e um elemento $x \in X$, e a existência de $k$ partições de $X$. Se separarmos em dois casos; o caso em que $x$ está sozinho em um subconjunto, então temos que os outros $n-1$ elementos devem ser distribuídos nos outros $k-1$ subconjuntos, consequentemente existirá $s(n-1, k-1)$ partições em que $x$ permanece sozinho. Por outro lado, fazer $k$ partições em que $x$ não fica sozinho é o mesmo que fazer $k$ partições de $X / x$, ou seja, de $s(n-1, k)$ maneiras, e escolher em qual dos $k$ subconjuntos fica $x$. Daí então:

$$
S(n, k)=\left\{\begin{array}{l}
n \\
k
\end{array}\right\}=S(n-1, k-1)+k S(n-1, k), \forall n>0, \forall k \leq n
$$

Tabela de valores com os números de Stirling de segunda espécie.

\begin{tabular}{|c|c|c|c|c|c|c|c|c|c|}
\hline$n$ & $S(n, 0)$ & $S(n, 1)$ & $S(n, 2)$ & $S(n, 3)$ & $S(n, 4)$ & $S(n, 5)$ & $S(n, 6)$ & $S(n, 7)$ & $S(n, 8)$ \\
\hline 0 & 1 & & & & & & & & \\
\hline 1 & 0 & 1 & & & & & & & \\
\hline 2 & 0 & 1 & 1 & & & & & & \\
\hline 3 & 0 & 1 & 3 & 1 & & & & & \\
\hline 4 & 0 & 1 & 7 & 6 & 1 & & & & \\
\hline 5 & 0 & 1 & 15 & 25 & 10 & 1 & & & \\
\hline 6 & 0 & 1 & 31 & 90 & 65 & 15 & 1 & & \\
\hline 7 & 0 & 1 & 63 & 301 & 350 & 140 & 21 & 1 & \\
\hline 8 & 0 & 1 & 127 & 966 & 1701 & 1050 & 266 & 28 & 1 \\
\hline
\end{tabular}

Note que para completar a linha $n=9$ é simples, pois já avaliamos a linha $n=8$, veja o esquema a seguir. 


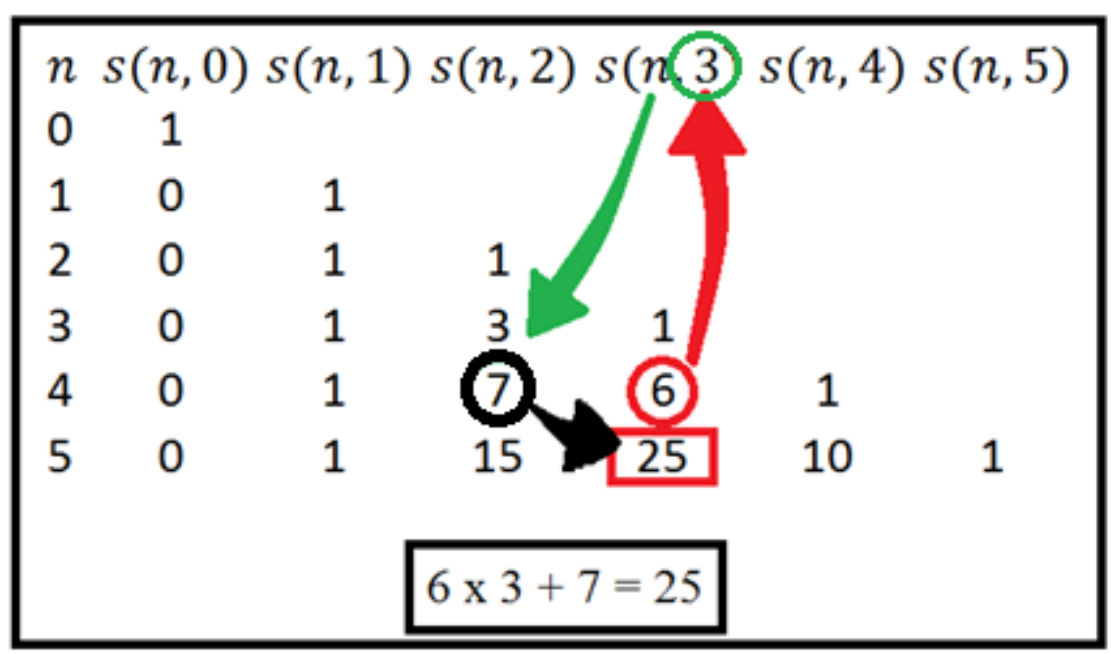

\section{Exemplo 14.}

Quantas partições há no conjunto $A=\{1,2,3,4,5\} ?$

Solução:

Basta somar a linha $k=5$

$$
1+15+25+10+1=52 \text { partições }
$$

O número de todas as partições de $\{1,2, \ldots, n\}$ em subconjuntos não vazios é dado pelo número de Bell.

$$
B(n)=\sum_{0}^{n} S(n, k)
$$

Relação de recorrência

$$
B(n+1)=\sum_{0}^{n}\left(\begin{array}{l}
n \\
j
\end{array}\right) B(j)
$$

pois assumindo que o elemento $n+1$ está em um subconjunto da partição com $n-k+1$ elementos, existem $\left(\begin{array}{c}n \\ n-k\end{array}\right)=\left(\begin{array}{l}n \\ k\end{array}\right)$ modos de escolher os elementos do mesmo subconjunto que contém $n+1$ e existem $B(j)$ maneiras de particionar o conjunto marcado pelos $k$ elementos restantes de $\{1,2, \ldots, n+1\}$. 
Os números de Bell podem ser obtidos facilmente, a partir do triângulo de Bell. Observe o esquema a seguir.

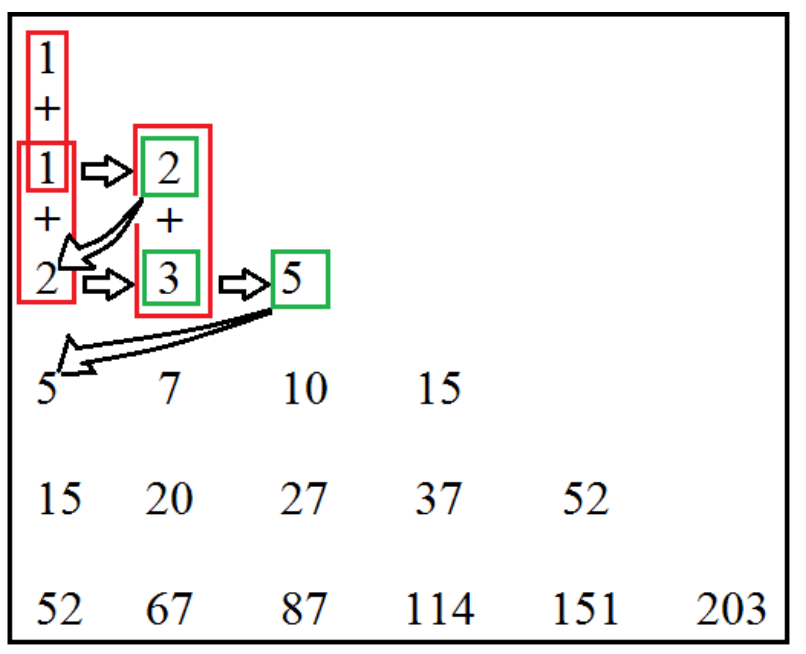

Para obter os números de Bell, basta tomar o último número de cada linha. Considere $B(0)=B(1)=1$

que pode ser organizado na tabela

\begin{tabular}{|c|c|c|c|c|c|c|c|c|c|}
\hline$n$ & 0 & 1 & 2 & 3 & 4 & 5 & 6 & 7 & 8 \\
\hline $\begin{array}{c}\text { Número } \\
\text { de } \\
\text { partições }\end{array}$ & 1 & 1 & 2 & 5 & 15 & 52 & 203 & 877 & 4140 \\
\hline
\end{tabular}

\subsection{OS LEMAS DE KAPLANSKY}

O primeiro lema de Kaplansky pode ser entendido como um método para contagem de $p$-subconjuntos, isto é, um subconjunto com $p$ elementos de $\{1,2,3, \ldots, n\}$ sem que haja números consecutivos.

\section{Exemplo 15.}

De quantos modos podemos formar um subconjunto com 4 elementos do conjunto $\{1,2,3,4,5,6,7,8,9\}$ sem que haja elementos consecutivos? 
Ao formar um subconjunto marcamos com 0 (zero) o elemento que não fará parte do subconjunto e com 1 (um) para o que fará parte, respeitando sua respectiva posição.

Veja alguns exemplos:

\begin{tabular}{|c|c|}
\hline Subconjunto & Notação binária representativa \\
\hline$\{1,4,6,9\}$ & 100101001 \\
\hline$\{2,4,7,9\}$ & 010100101 \\
\hline$\{1,3,5,8\}$ & 101010010 \\
\hline$\{1,2,5,8\}$ & 110010010 \\
\hline
\end{tabular}

Dos exemplos anteriores, apenas o último não satisfaz as condições do problema, pois nesse os elementos 1 e 2 aparecem no mesmo subconjunto. Dessa forma, queremos encontrar locais para inserir o algarismo 1 entre os zeros.

Observe que podemos inserir o algarismo 1 em seis locais diferentes. Sendo assim, a solução do problema é escolher 4 dos 6 lugares para a ocupação do algarismo 1, ou seja, $\left(\begin{array}{l}6 \\ 4\end{array}\right)=\frac{6 !}{4 !(6-4) !}=15$ maneiras para formar 4-subconjuntos de $\{1,2,3,4,5,6,7,8,9\}$ sem elementos consecutivos.

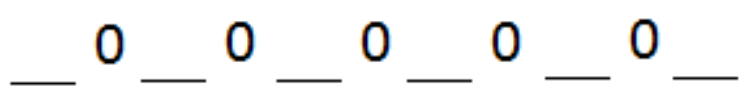

No caso geral, temos $p$ algarismos 1 (um) e $n-p$ algarismos 0 (zero). Temos um modo de colocar os algarismo 0 (zero) e $\left(\begin{array}{c}n-p+1 \\ p\end{array}\right)$ de colocarmos o algarismo 1. Sendo assim, o número de $p$-subconjuntos de $\{1,2,3, \ldots, n\}$ sem que haja elementos consecutivos é dado pela fórmula:

$$
f(n, p)=\left(\begin{array}{c}
n-p+1 \\
p
\end{array}\right)
$$

Suponha agora que os elementos do conjunto $\{1,2,3, \ldots, n\}$ tenham sido colocados em círculo. Dessa forma, os algarismos 1 e $n$ são consecutivos e não devem aparecer em um mesmo subconjunto. De quantos modos podemos formar $p$-subconjuntos de $\{1,2,3, \ldots, n\}$ no qual não tenha elementos consecutivos? 


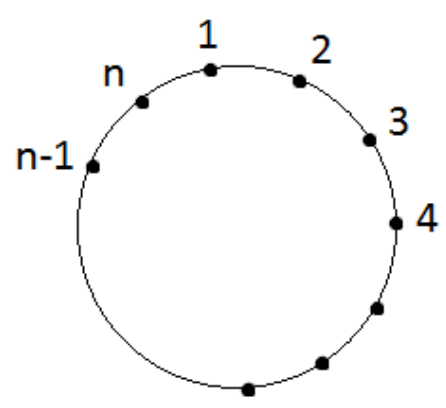

O total de subconjuntos será a soma entre os subconjuntos em que o elemento 1 figura e os subconjuntos em que o elemento 1 não figura.

$1^{\circ}$ caso: (Elemento 1 figura) para formá-los devemos escolher $p-1$ elementos de $\{3,4,5, \ldots, n-1\}$, pois se 1 figura, 2 e o $n$ não podem figurar.

$$
f(n-3, p-1)=\left(\begin{array}{c}
n-3-(p-1)+1 \\
p-1
\end{array}\right)=\left(\begin{array}{c}
n-p-1 \\
p-1
\end{array}\right)
$$

$2^{\circ}$ caso: (Elemento 1 não figura) para formá-los escolhemos $p$ elementos (não consecutivos) de $\{2,3, \ldots, n\}$, que podem ser obtidos por $f(n-1, p)=\left(\begin{array}{c}n-1-p+1 \\ p\end{array}\right)=$ $\left(\begin{array}{c}n-p \\ p\end{array}\right)$ modos. Portanto, a solução é dada por:

$$
\begin{gathered}
f(n-3, p-1)+f(n-1, p)=\left(\begin{array}{c}
n-p-1 \\
p-1
\end{array}\right)+\left(\begin{array}{c}
n-p \\
p
\end{array}\right) \\
=\frac{(n-p-1) !}{(p-1) !(n-2 p) !}+\frac{(n-p) !}{p !(n-2 p) !} \\
=\frac{(n-p-1) ! p+(n-p) !}{p !(n-2 p) !} \\
=(n-p-1) ! \frac{p+(n-p)}{p !(n-2 p) !}=n \frac{(n-p-1) !}{p !(n-2 p) !} \\
=\frac{n}{n-p} \frac{(n-p) !}{p !(n-2 p) !}=\frac{n}{n-p}\left(\begin{array}{c}
n-p \\
p
\end{array}\right)
\end{gathered}
$$

Assim temos o segundo lema de Kaplansky, em que o número de p-subconjuntos de $\{1,2,3, \ldots, n\}$ nos quais não há números consecutivos e, considerando 1 e $n$ como consecutivos iguais a 


$$
g(n, p)=\frac{n}{n-p}\left(\begin{array}{c}
n-p \\
p
\end{array}\right)
$$

\subsection{APLICAÇÕES PARA A SEQUÊNCIA DE FIBONACCI}

$\mathrm{O}$ número de subconjuntos vazios e não vazios de $\{1,2,3, \ldots, n\}$ contendo elementos não consecutivos é dado por $F_{n+2}$ em que $F_{n+2}$ caracteriza um número da sequência de Fibonacci, a saber, $F_{1}=1, F_{2}=1, F_{3}=2, F_{4}=3, F_{5}=5, F_{n}=F_{n-1}+F_{n-2}$.

\section{Exemplo 16.}

O número de subconjuntos vazios e não vazios com elementos não consecutivos de $\{1,2,3,4\}$ é $F_{4+2}=F_{6}=8$. Sendo eles

$$
\emptyset ;\{1\} ;\{2\} ;\{3\} ;\{4\} ;\{1,3\} ;\{1,4\} ;\{2,4\}
$$

Prova por indução.

Hipótese de indução:

Para $j=1,2, \ldots, n$ o número de subconjuntos $S \subset\{1,2, \ldots, j\}$ sem elementos consecutivos é $F_{j+2}$.

Tese de indução:

$S \subset\{1,2, \ldots, n, n+1\}, S$ não contém elementos consecutivos.

Caso 1: $n+1 \notin S$

$S \subset\{1,2, \ldots, n\}$ e o número de subconjuntos é $F_{n+2}$

Caso 2: $n+1 \in S \Rightarrow n \notin S$

$S \backslash\{n+1\} \subset\{1,2, \ldots, n-1\}$ número de subconjuntos $F_{n-1+2}=F_{n+1}$

$$
\text { Total } F_{n+1}+F_{n+2}=F_{n+3}=F_{(n+1)+2}
$$




\section{Exemplo 17.}

Supondo que uma pessoa, ao subir uma escada, consiga, no máximo, pular um degrau por vez. Dessa forma de quantas maneiras pode-se subir uma escada com $n$ degraus?

- Para $n=1$, uma maneira de se elevar do piso $\alpha$ para o piso $\beta$.

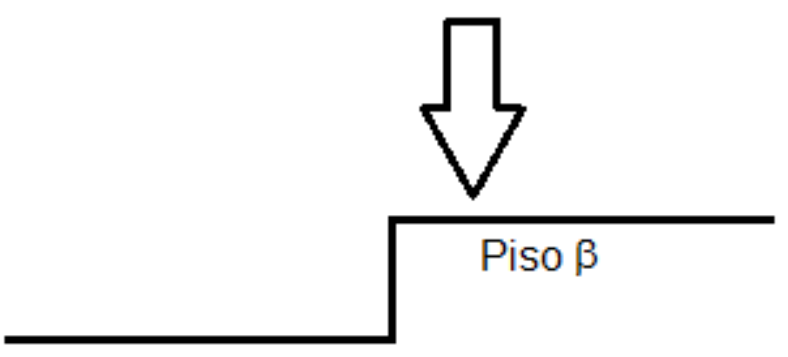

Piso $\alpha$

- Para $n=2$, há 2 formas.

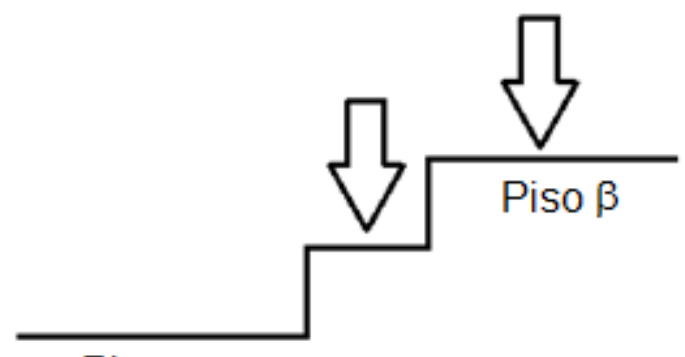

Piso $\alpha$

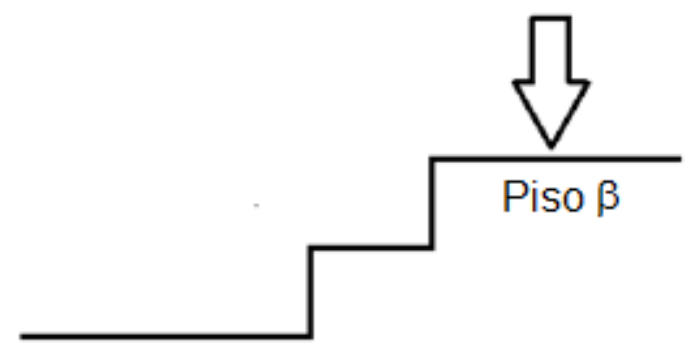

Piso $\alpha$

- Para $n=3$, há 3 formas.
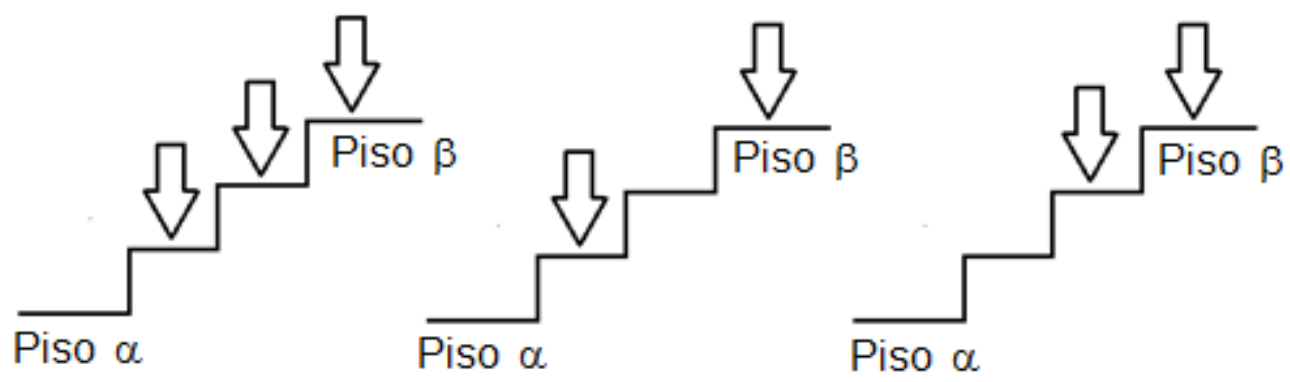

- Para $n=4$, há 5 formas. 

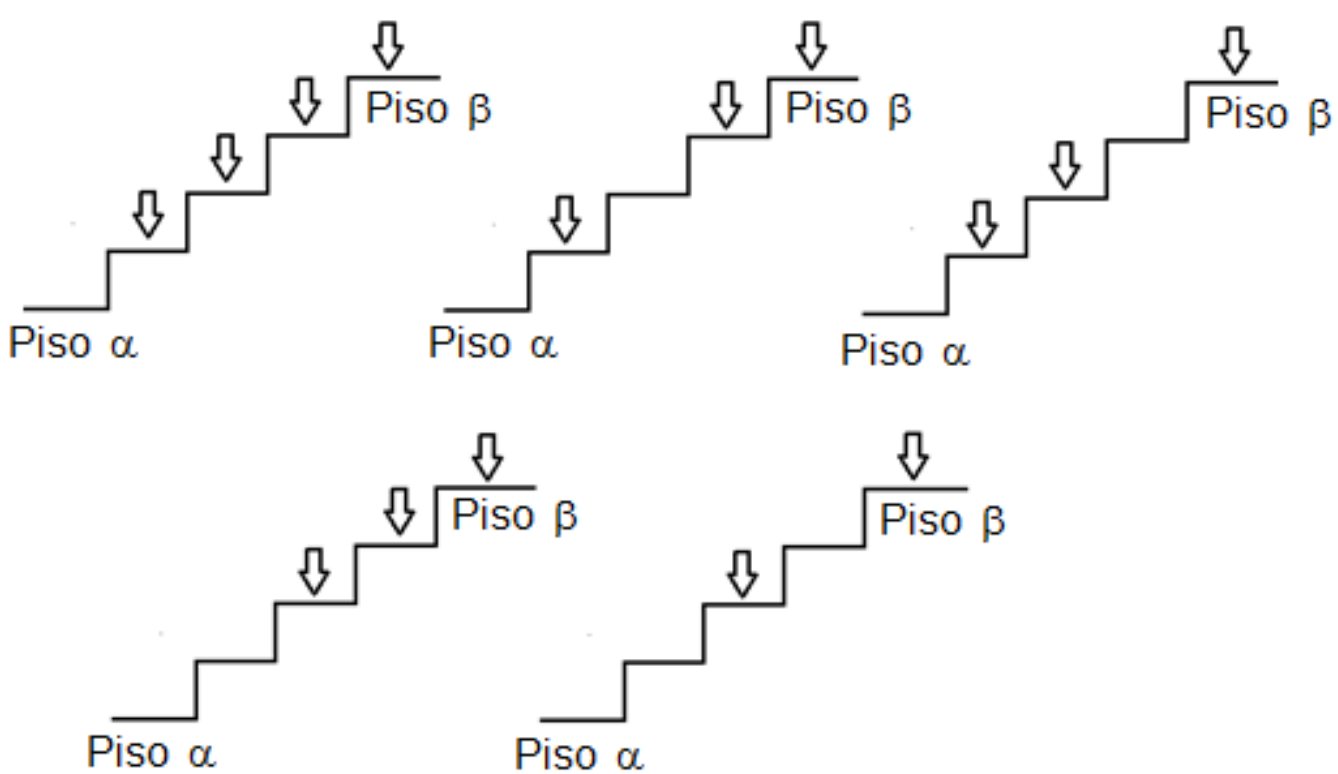

Seja $f_{n}$ o número de maneiras de subir a escada. Observamos que $f_{1}=1, f_{2}=2, f_{3}=$ $3, f_{4}=5$, que são respectivamente iguais aos números de Fibonacci $F_{2}, F_{3}, F_{4}, F_{5}$.

Provemos que a sequência $f_{n}$ está definida sobre a mesma recorrência que os números de Fibonacci. Dividindo o problema em dois casos, temos,

Primeiro caso: O primeiro degrau é usado, então restam $n-1$ degraus, logo, por definição existem $f_{n-1}$ formas de usá-los.

Segundo caso: A subida parte do segundo degrau, então restam $n-2$ degraus, logo, por definição existem $f_{n-2}$ formas de usá-los.

Por não haver outra maneira de iniciar a subida temos que $f_{n}=f_{n-1}+f_{n-2}$, ou seja, a sequência respeita a recorrência de Fibonacci. Porém, $\operatorname{como} f_{n} \neq F_{n}$, é necessário antecipar um elemento da sequência de Fibonacci para termos a identidade. Assim:

$$
f_{n}=F_{n+1}
$$




\section{CONSIDERAÇÕES FINAIS}

Concluímos, por meio das abordagens expostas, que o tema Análise Combinatória não se limita apenas ao estudo de arranjos, permutações e combinações. Cabe aos professores e alunos explorarem o tema ampliando o conhecimento, adquirindo novas técnicas e procedimentos para que, dessa forma, seja possível proporcionar soluções a uma maior variedade de contextos ou problemas.

Cabe ainda recomendar a existência de outros números e procedimentos que podem virar tema de estudo a professores e alunos, como por exemplo, os números de Lucas, os números Harmônicos, o Princípio das Gavetas de Dirichlet também conhecido como Princípio da Casa dos Pombos. 


\section{Referências}

[1] BRASIL.Parâmetros Curriculares De Matemática. Secretaria De Ensino Médio. Brasília: MEC/SEF, Volumes 1 e 3. 1999, 2000.

[2] CDCC - USP. Análise Combinatória e Probabilidade, genética e probabilidade. Disponível em: http://www.cdcc.usp.br/exper/medio/matematica/matematica_medio/4_genetica_e _combinatoria_p.pdf. Acesso em: 10.01.2014.

[3] GOMES, Carlos A. $\mathbf{E}$ as funções sobrejetoras?Disponível em: http://www.olimpiada.ccet.ufrn.br/wp-content/uploads/2013/08/nota_aula_05.pdf. Acesso em 14.03.2014.

[4] GRAHAM, R. L.; KNUTH, D. E. e PATASHNIK, O. Concrete Mathematics, A Foundation for Computer Science. Pearson Education, 1994.

[5] MORGADO, A.C.et al. - Análise Combinatória e Probabilidade. Coleção do Professor de Matemática. Rio de Janeiro: SBM, 2004.

[6] OLIVEIRA, Fabrício. A. et al.Um estudo das permutações caóticas. Disponível em:http://www.portal.famat.ufu.br/sites/famat.ufu.br/files/Anexos/Bookpage/famat _revista_13_sala_de_aula2.pdf. Acesso em: 18.09.2014.

[7] ROSEN, Kenneth H. Discrete Mathematics and Its Applications. McGraw Hill, 1995.

[8] SANTOS, José Plínio, MELLO, Margarida P., MURARI, Idani T. C. Introdução à Análise Combinatória. Rio de Janeiro:Ed. Ciência Moderna, 2007.

[9] Função totiente de Euler. Disponível em: http://pt.wikipedia.org/wiki/Fun\%C3\%A7\%C3\%A3o_totiente_de_Euler. Acesso em: 30.10 .2014 